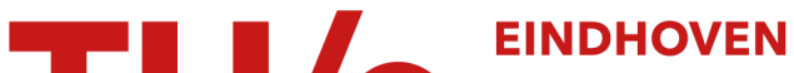 \\ UNIVERSITY OF \\ TECHNOLOGY
}

\section{Tracking control for nonlinear networked control systems}

Citation for published version (APA):

Postoyan, R., Wouw, van de, N., Nesic, D., \& Heemels, W. P. M. H. (2014). Tracking control for nonlinear networked control systems. IEEE Transactions on Automatic Control, 59(6), 1539-1554.

https://doi.org/10.1109/TAC.2014.2308598

DOI:

10.1109/TAC.2014.2308598

Document status and date:

Published: 01/01/2014

\section{Document Version:}

Publisher's PDF, also known as Version of Record (includes final page, issue and volume numbers)

\section{Please check the document version of this publication:}

- A submitted manuscript is the version of the article upon submission and before peer-review. There can be important differences between the submitted version and the official published version of record. People interested in the research are advised to contact the author for the final version of the publication, or visit the $\mathrm{DOI}$ to the publisher's website.

- The final author version and the galley proof are versions of the publication after peer review.

- The final published version features the final layout of the paper including the volume, issue and page numbers.

Link to publication

\section{General rights}

Copyright and moral rights for the publications made accessible in the public portal are retained by the authors and/or other copyright owners and it is a condition of accessing publications that users recognise and abide by the legal requirements associated with these rights.

- Users may download and print one copy of any publication from the public portal for the purpose of private study or research.

- You may not further distribute the material or use it for any profit-making activity or commercial gain

- You may freely distribute the URL identifying the publication in the public portal.

If the publication is distributed under the terms of Article 25fa of the Dutch Copyright Act, indicated by the "Taverne" license above, please follow below link for the End User Agreement:

www.tue.nl/taverne

Take down policy

If you believe that this document breaches copyright please contact us at:

openaccess@tue.nl

providing details and we will investigate your claim. 


\title{
Tracking Control for Nonlinear Networked Control Systems
}

\author{
Romain Postoyan, Nathan van de Wouw, Dragan Nešić, Fellow, IEEE, and \\ W. P. Maurice H. Heemels, Senior Member, IEEE
}

\begin{abstract}
We investigate the tracking control of nonlinear networked control systems (NCS) affected by disturbances. We consider a general scenario in which the network is used to ensure the communication between the controller, the plant and the reference system generating the desired trajectory to be tracked. The communication constraints induce non-vanishing errors (in general) on the feedforward term and the output of the reference system, which affect the convergence of the tracking error. As a consequence, available results on the stabilization of equilibrium points for NCS are not applicable. Therefore, we develop an appropriate hybrid model and we give sufficient conditions on the closed-loop system, the communication protocol and an explicit bound on the maximum allowable transmission interval guaranteeing that the tracking error converges to the origin up to some errors due to both the external disturbances and the aforementioned nonvanishing network-induced errors. The results cover a large class of the so-called uniformly globally asymptotically stable protocols which include the well-known round-robin and try-once-discard protocols. We also introduce a new dynamic protocol suitable for tracking control. Finally, we show that our approach can be used to derive new results for the observer design problem for NCS. It has to be emphasized that the approach is also new for the particular case of sampled-data systems.
\end{abstract}

Index Terms-Hybrid systems, networked control systems, observers, sampled-data, tracking control.

\section{INTRODUCTION}

$\mathbf{N}$ ETWORKED control systems (NCS) have received considerable research interest these last decades. This is justified by the fact that, nowadays, controllers often communicate with the plant via a network which may be used for other tasks as well. This implementation offers great advantages

Manuscript received August 23, 2012; revised June 5, 2013 and November 27, 2013; accepted February 5, 2014. Date of publication February 26, 2014; date of current version May 20, 2014. A preliminary version of this work was presented at the 51st IEEE Conference on Decision and Control [1]. This work was supported by the European 7th Framework Network of Excellence "Highly-complex and networked control systems" (HYCON2) under grant 257462, the Australian Research Council under the Discovery Projects and Future Fellowship schemes and the Innovational Research Incentives Scheme under the VICI grant "Wireless control systems: A new frontier in automation" (11382) awarded by NWO (The Netherlands Organization for Scientific Research) and STW (Dutch Science Foundation). Recommended by Associate Editor G. E. Dullerud.

R. Postoyan is with the Université de Lorraine, CRAN, UMR 7039 and the CNRS, CRAN, UMR 7039, France (e-mail: romain.postoyan@univ-lorraine.fr).

N. van de Wouw and W. P. M. H. Heemels are with the Department of Mechanical Engineering, Eindhoven University of Technology, Eindhoven, The Netherlands (e-mail: n.v.d.wouw@tue.nl; m.heemels@tue.nl).

D. Nešić is with the Department of Electrical and Electronic Engineering, the University of Melbourne, Parkville, VIC 3010, Australia (e-mail: dnesic@ unimelb.edu.au).

Color versions of one or more of the figures in this paper are available online at http://ieeexplore.ieee.org.

Digital Object Identifier 10.1109/TAC.2014.2308598 over classical point-to-point connections in terms of cost, flexibility and ease of maintenance. On the other hand, it requires the development of appropriate control strategies to guarantee the desired stability properties under the communication constraints caused by the use of the network. Most available results on NCS concentrate on the stabilization of equilibrium points (see for example [2]-[6]), while very few studies address the tracking control of NCS, see [7]-[9], although this problem is fundamental in control theory. The latter references have shown that tracking control exhibits specific difficulties which are due to the use of the communication channel and which are absent when considering the stabilization of an equilibrium point. Indeed, tracking controllers are often composed of a feedback term (to ensure the convergence to the desired solution) and a feedforward term (which induces the desired solution in the closed-loop system). The authors of [7]-[9] have shown that the errors induced by the network on the feedforward term lead to approximate tracking. Similarly, the fact that the reference signals are transmitted via the communication channel may also be a source of errors that obstruct the convergence of the tracking error to zero.

The main purpose of the present paper is to propose a method to design controllers which achieve a state tracking objective for NCS affected by exogenous perturbations. The reference to be tracked can either be given as a reference trajectory or as the states of a reference system as in the master-slave synchronization problem. We follow an emulation-like approach as in [2]-[6] which consists in first designing a controller that solves the problem in the absence of communication constraints. Afterwards, we implement the controller over a network and study the conditions that preserve the tracking property up to some errors caused by the network. We consider a general scenario where the channel is used to ensure the communication between the controller, the plant and the reference system. This allows us to encompass the architectures studied in [7]-[9] as particular cases and to investigate a rich class of new ones. At each transmission instant, the network is such that only a single node (i.e. a group of sensors or actuators) is granted access to the network according to a rule called scheduling protocol. The class of protocols we consider includes the round-robin (RR) protocol, the try-once-discard (TOD) protocol [6] and more generally the protocols which are Lyapunov uniformly globally asymptotically stable (UGAS) as defined in [5]. We also propose a new dynamic protocol for tracking control which may ensure improved performance compared to the RR and TOD protocols. In comparison to [7]-[9], we consider nonlinear systems (as opposed to linear systems) and we study the 
effect of sampling and scheduling (as opposed to sampling and delays or quantization, although we believe that the framework laid down in this paper allows extensions in these directions by exploiting the ideas from [3], [10] for instance).

We present a new hybrid model using the formalism of [11] to study the tracking control of NCS which is general enough to describe the setups of [7]-[9] and to represent various new architectures as mentioned above. It relies on the choice of a specific set of coordinates which facilitates the analysis afterwards. Next we state sufficient conditions on the closedloop system and we provide an explicit and easy-to-use bound on the maximum allowable transmission interval (MATI) to ensure that the tracking error converges to the origin up to some errors due to the external perturbations, as expected, but also due to the aforementioned network-induced errors. These additional errors constitute an essential difference with the scenario where an equilibrium point has to be stabilized and they induce supplementary technical difficulties. Indeed, the stability analysis is based on the construction of a hybrid Lyapunov function inspired by [2], which exhibits the feature of potentially increasing at jumps (as opposed to [2]). We then provide guidelines on how to implement the controller and to design the scheduling protocol to reduce the impact of the nonvanishing network-induced errors on the tracking accuracy.

Building upon the analogies which exist between masterslave synchronization and observer design [12], we also derive new results for the observer design problem for NCS. Compared to [13], [14], we rely on a Lyapunov-based analysis (as opposed to trajectory-based arguments) and we provide a new bound on the MATI. In addition, we envision an emulation procedure similar to [15] which allows us to relax some of the assumptions of [13], [14] for the considered class of systems. It has to be noticed that we focus on a more general class of observers than that in [15] and that we propose a different stability analysis as well as a different MATI bound. Overall, we would like to emphasize that the presented results are new in the context of sampled-data systems (with non-uniform sampling), in which case the scheduling protocol grants access to all nodes at each transmission instant.

The paper is organized as follows. Preliminaries are presented in Section II. The tracking control problem is formalized in Section III. Next, we propose a suitable NCS model in Section IV and the assumptions we adopt are given in Section V. The main stability results are stated in Section VI. In Section VII, we give examples of protocols suitable in the scope of tracking. The application of the derived results to the observer design problem for NCS is presented in Section VIII. Examples are provided in Section IX. All the proofs are given in the Appendix.

\section{PREliminaries}

Let $\mathbb{R}:=(-\infty, \infty), \mathbb{R}_{\geq 0}:=[0, \infty), \mathbb{R}_{>0}:=(0, \infty), \mathbb{Z}_{\geq 0}:=$ $\{0,1,2, \ldots\}$, and $\mathbb{Z}_{>0}:=\{1,2, \ldots\}$. A function $\gamma: \mathbb{R}_{\geq 0} \rightarrow \mathbb{R}_{\geq 0}$ is of class $\mathcal{K}$ if it is continuous, zero at zero and strictly increasing, and it is of class $\mathcal{K}_{\infty}$ if in addition it is unbounded. $\mathrm{A}$ continuous function $\gamma: \mathbb{R}_{>0}^{2} \longrightarrow \mathbb{R}_{>0}$ is of class $\mathcal{K} \mathcal{L}$ if for each $t \in \mathbb{R}_{\geq 0}, \gamma(\cdot, t)$ is of class $\mathcal{K}$, and, for each $s \in \mathbb{R}_{>0}, \gamma(s, \cdot)$ is decreasing to zero. Additionally, a function $\beta: \mathbb{R}_{\geq 0}^{3} \rightarrow \mathbb{R}_{\geq 0}$ is of class $\mathcal{K} \mathcal{L} \mathcal{L}$, if $\beta(\cdot, \cdot, t) \in \mathcal{K} \mathcal{L}$ and $\beta(\cdot, t, \cdot) \in \mathcal{K} \mathcal{L}$ for any $t \in \mathbb{R}_{\geq 0}$. For $x \in \mathbb{R}^{n}$ and $y \in \mathbb{R}^{m}$, the notation $(x, y)$ stands for $\left[x^{\mathrm{T}}, y^{\mathrm{T}}\right]^{\mathrm{T}}$. We use $\mathbb{I}_{n}$ to denote the identity matrix of dimension $n$ and $\operatorname{diag}\left(A_{1}, A_{2}\right)$ to denote the block diagonal matrix made of the square matrices $A_{1}$ and $A_{2}$. For $(t, j),(s, k) \in \mathbb{R} \times \mathbb{Z}_{\geq 0}$, we write $(t, j) \preceq(s, k)$ if $t+j \leq s+k$.

We will study hybrid systems of the form below using the formalism of [16], [17]

$$
\dot{x}=f(x, w) \text { for } x \in C, \quad x^{+}=g(x, w) \text { for } x \in D
$$

where $x \in \mathbb{R}^{n}$ is the state, $w \in \mathbb{R}^{m}$ is the input, $f$ is the flow map, $g$ is the jump map, $C$ is the flow set and $D$ is the jump set. We assume that $C$ and $D$ are closed subsets of $\mathbb{R}^{n}$ and that $f$ and $g$ are respectively continuous on $C$ and on $D$. A subset $E \subset$ $\mathbb{R}_{\geq 0} \times \mathbb{Z}_{\geq 0}$ is a hybrid time domain if for all $(T, J) \in E, E \cap$ $([0, T] \times\{0, \ldots, J\})=\bigcup_{j \in\{0,1, \ldots, J-1\}}\left(\left[t_{j}, t_{j+1}\right], j\right)$ for some finite sequence of times $0=t_{0} \leq t_{1} \leq \ldots \leq t_{J}$. A function $w$ : $E \rightarrow \mathbb{R}^{m}$ is a hybrid input if $E$ is a hybrid time domain and if $w(\cdot, j)$ is Lebesgue measurable and locally essentially bounded for each $j$. A function $x: E \rightarrow \mathbb{R}^{n}$ is a hybrid arc if $E$ is a hybrid time domain and if $x(\cdot, j)$ is locally absolutely continuous for each $j$. The hybrid arc $x: \operatorname{dom} x \rightarrow \mathbb{R}^{n}$ and the hybrid input $w: \operatorname{dom} w \rightarrow \mathbb{R}^{m}$ is a solution pair to (1) if: i) $\operatorname{dom} x=\operatorname{dom} w$ and $x(0,0) \in C \cup D$; ii) for any $j \in \mathbb{Z}_{\geq 0}, x(t, j) \in C$ and $d / d t$ $x(t, j)=f(x(t, j), w(t, j))$ for almost all $t \in \mathcal{I}^{j}$ where $\mathcal{I}^{j}=\{t$ : $(t, j) \in \operatorname{dom} x\}$; iii) for every $(t, j) \in \operatorname{dom} x$ such that $(t, j+$ $1) \in \operatorname{dom} x, x(t, j) \in D$ and $x(t, j+1)=g(x(t, j), w(t, j))$. A solution pair $(x, u)$ to (1) is maximal if it cannot be extended, and it is complete if $\operatorname{dom} x$ is unbounded. Let $w$ be a hybrid signal with $(0,0)$ as initial hybrid time, we define $\|w\|_{(t, j)}:=\max \left\{\begin{array}{l}\text { ess.sup } \\ \left(t^{\prime}, j^{\prime}\right) \in \operatorname{dom} w \backslash \Gamma(w),(0,0) \prec\left(t^{\prime}, j^{\prime}\right) \prec(t, j)\end{array}\left|w\left(t^{\prime}, j^{\prime}\right)\right|\right.$, $\left.\sup \quad \sup \left|w\left(t^{\prime}, j^{\prime}\right)\right|\right\}$ where $\Gamma(w)$ is the $\left(t^{\prime}, j^{\prime}\right) \in \Gamma(w),(0,0) \preceq\left(t^{\prime}, j^{\prime}\right) \preceq(t, j)$

set of all $\left(t^{\prime}, j^{\prime}\right) \in \operatorname{dom} w$ such that $\left(t^{\prime}, j^{\prime}+1\right) \in \operatorname{dom} w$.

\section{Problem Statement}

\section{A. The Tracking Problem}

Consider the nonlinear plant model

$$
\dot{x}_{p}=\mathbf{f}_{p}\left(x_{p}, u, w_{p}\right), \quad y_{p}=\mathbf{g}_{p}\left(x_{p}\right)
$$

where $x_{p} \in \mathbb{R}^{n_{x}}$ is the state, $u \in \mathbb{R}^{n_{u}}$ the control input, $y_{p} \in$ $\mathbb{R}^{n_{y}}$ the measured output and $w_{p} \in \mathbb{R}^{n_{w_{p}}}$ is an external perturbation. The reference $x_{d}$ that system (2) has to track is given by the solution to

$$
\dot{x}_{d}=\mathbf{f}_{p}\left(x_{d}, u_{f f}, w_{d}\right), \quad y_{d}=\mathbf{g}_{p}\left(x_{d}\right)
$$

where $u_{f f} \in \mathbb{R}^{n_{u}}$ is the (feedforward) input, $y_{d} \in \mathbb{R}^{n_{y}}$ denotes the measured output and $w_{d} \in \mathbb{R}^{n_{w_{d}}}$ is a vector of external disturbances. When $x_{d}$ is a given reference trajectory, $w_{d}$ may model the uncertainty on the feedforward term $u_{f f}$ when its exact expression is not available. System (3) may also model a master system that the plant (2) has to synchronize with. In this scenario, the master system (3) may be affected by external disturbances which justifies the presence of $w_{d}$ in (3). We assume that the reference system (3) has a unique solution for any 


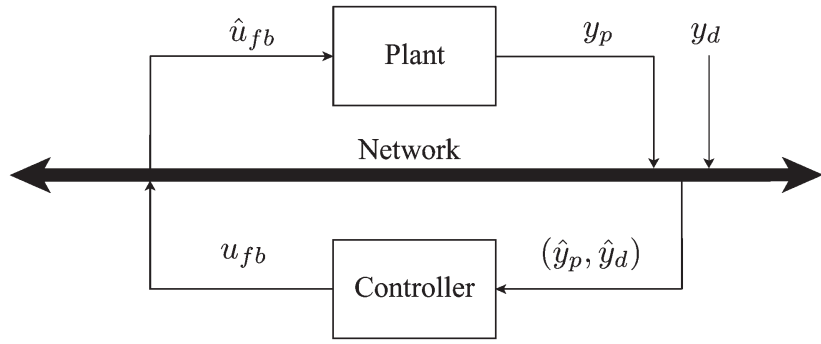

Fig. 1. Block diagram of the tracking control of NCS studied in [7], [9].

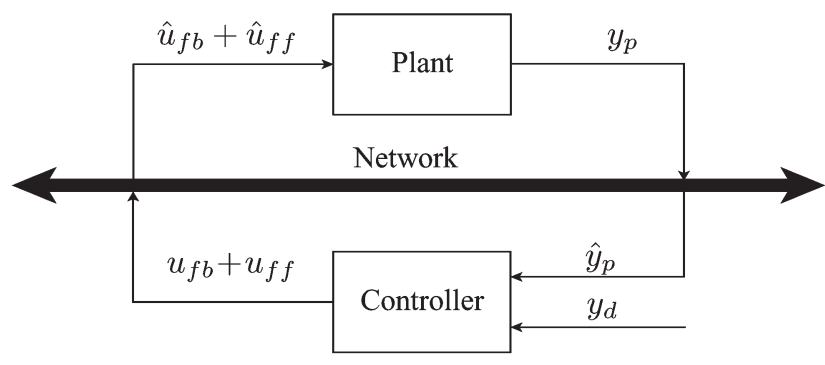

Fig. 2. Block diagram of the tracking control of NCS studied in [8].

initial condition $x_{d}(0)$ and any inputs $u_{f f}$ and $w_{d}$ of interest. Both $u_{f f}$ and $y_{d}$ are available for the purpose of control.

We consider the following controller decomposition

$$
u=u_{f b}+u_{f f}
$$

where the feedforward term $u_{f f}$ comes from (3) and the feedback term $u_{f b}$ is the output of the dynamic controller given by

$$
\dot{x}_{c}=\mathbf{f}_{c}\left(x_{c}, y_{p}, y_{d}, w_{c}\right), \quad u_{f b}=\mathbf{g}_{c}\left(x_{c}\right)
$$

where $x_{c} \in \mathbb{R}^{n_{x_{c}}}$ is the controller state and $w_{c} \in \mathbb{R}^{n_{w_{c}}}$ is a vector of perturbations which may affect the controller dynamics.

\section{B. Controller Implementation Over the Network}

We investigate the scenario where a network is used to ensure the communication between the plant sensors and the controller and between the controller and the plant actuators. We also allow for the case where the communication channel is used to transmit the output and the input of the reference system (3), i.e. $y_{d}$ and $u_{f f}$. We consider a general setting because we can then capture, in a unified manner, specific scenarios in which the network is only used to realize some relevant subsets of the aforementioned communications, such as e.g. the cases in:

- [7], [9] where the reference and plant outputs, $y_{d}$ and $y_{p}$ respectively, are sent together to the controller and $u_{f f}$ is not transmitted, see Fig. 1.

- [8] where the output $y_{d}$ is directly available to the controller and $u_{f f}$ is generated by the controller (note that $y_{d}=x_{d}$ in [8]), see Fig. 2.

Our approach also allows us to study the scenario depicted in Fig. 3 for instance, where the reference output $y_{d}$ and the feedforward term $u_{f f}$ are transmitted via the network. In that case, it is reasonable to set up the network in such a way that the feedforward term $u_{f f}$ is directly transmitted to the plant actuators.

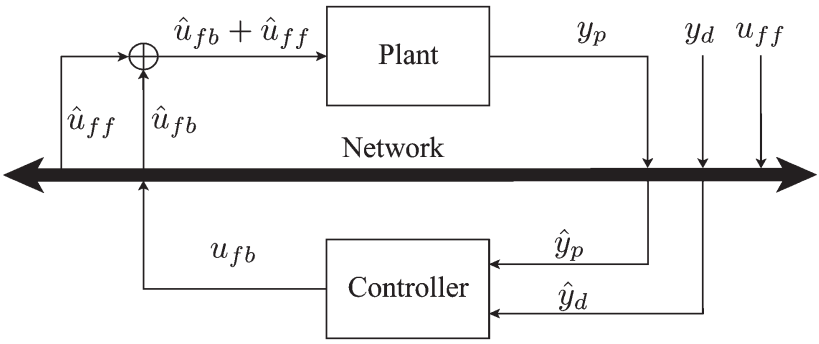

Fig. 3. Block diagram of the tracking control of NCS when $u_{f f}$ is sent by the reference system.

The sensors and the actuators of the plant (2) and of the reference system (3) are grouped into $\ell$ nodes (depending on their spatial location) which are connected to the network. At each transmission instant $t_{i}, i \in \mathbb{Z}_{>0}$, only one node is granted access to the network by the scheduling protocol. The transmission sequence $\left\{t_{i}\right\}_{i \in \mathbb{Z}_{>0}}$ is such that $v \leq t_{i}-t_{i-1} \leq \tau^{*}$ for $i \in \mathbb{Z}_{>0}$, where $\tau^{*} \in \mathbb{R}_{>0}$ is the MATI and $v$ is the lower bound on the minimum achievable transmission interval given by the hardware constraints (see [4]). Notice that the transmission intervals $t_{i}-t_{i-1}$ may be time-varying and uncertain.

The plant (2) no longer receives $u=u_{f b}+u_{f f}$ but $\hat{u}=$ $\hat{u}_{f b}+\hat{u}_{f f}$ which is generated from the most recently transmitted feedback and feedforward terms. We distinguish the feedback term $u_{f b}$ from the feedforward term $u_{f f}$ because these may be transmitted via distinct nodes (see Fig. 3 for instance). The dynamics of the plant now becomes

$$
\begin{aligned}
\dot{x}_{p} & =\mathbf{f}_{p}\left(x_{p}, \hat{u}_{f b}+\hat{u}_{f f}, w_{p}\right) \quad t \in\left[t_{i-1}, t_{i}\right] \\
y_{p} & =\mathbf{g}_{p}\left(x_{p}\right) .
\end{aligned}
$$

Similarly, the controller (5) no longer receives $y_{p}$ and $y_{d}$ but their networked versions $\hat{y}_{p}$ and $\hat{y}_{d}$

$$
\begin{aligned}
\dot{x}_{c} & =\mathbf{f}_{c}\left(x_{c}, \hat{y}_{p}, \hat{y}_{d}, w_{c}\right) \quad t \in\left[t_{i-1}, t_{i}\right] \\
u_{f b} & =\mathbf{g}_{c}\left(x_{c}\right) .
\end{aligned}
$$

The variables $\hat{u}_{f b}, \hat{u}_{f f}, \hat{y}_{p}, \hat{y}_{d}$ have the following dynamics:

$$
\left.\begin{array}{rl}
\dot{\hat{u}}_{f b} & =\hat{\mathbf{f}}_{f b}\left(x_{p}, x_{c}, x_{d}, \hat{y}_{p}, \hat{y}_{d}, \hat{u}_{f b}, \hat{u}_{f f}\right) \\
\dot{\hat{u}}_{f f} & =\hat{\mathbf{f}}_{f f}\left(x_{p}, x_{c}, x_{d}, \hat{y}_{p}, \hat{y}_{d}, \hat{u}_{f b}, \hat{u}_{f f}\right) \\
\dot{\hat{y}}_{p} & =\hat{\mathbf{f}}_{p}\left(x_{p}, x_{c}, x_{d}, \hat{y}_{p}, \hat{y}_{d}, \hat{u}_{f b}, \hat{u}_{f f}\right) \\
\dot{\hat{y}}_{d} & =\hat{\mathbf{f}}_{d}\left(x_{p}, x_{c}, x_{d}, \hat{y}_{p}, \hat{y}_{d}, \hat{u}_{f b}, \hat{u}_{f f}\right)
\end{array}\right\} t \in\left[t_{i-1}, t_{i}\right]
$$

and

$$
\begin{aligned}
\hat{u}_{f b}\left(t_{i}^{+}\right) & =u_{f b}\left(t_{i}\right)+\mathbf{h}_{f b}\left(i, e_{p}\left(t_{i}\right), e_{d}\left(t_{i}\right), e_{f b}\left(t_{i}\right), e_{f f}\left(t_{i}\right)\right) \\
\hat{u}_{f f}\left(t_{i}^{+}\right) & =u_{f f}\left(t_{i}\right)+\mathbf{h}_{f f}\left(i, e_{p}\left(t_{i}\right), e_{d}\left(t_{i}\right), e_{f b}\left(t_{i}\right), e_{f f}\left(t_{i}\right)\right) \\
\hat{y}_{p}\left(t_{i}^{+}\right) & =y_{p}\left(t_{i}\right)+\mathbf{h}_{p}\left(i, e_{p}\left(t_{i}\right), e_{d}\left(t_{i}\right), e_{f b}\left(t_{i}\right), e_{f f}\left(t_{i}\right)\right) \\
\hat{y}_{d}\left(t_{i}^{+}\right) & =y_{d}\left(t_{i}\right)+\mathbf{h}_{d}\left(i, e_{p}\left(t_{i}\right), e_{d}\left(t_{i}\right), e_{f b}\left(t_{i}\right), e_{f f}\left(t_{i}\right)\right)
\end{aligned}
$$

where $e_{f b}:=\hat{u}_{f b}-u_{f b} \in \mathbb{R}^{n_{e_{u}}}, e_{f f}:=\hat{u}_{f f}-u_{f f} \in \mathbb{R}^{n_{e_{u}}}$, $e_{p}:=\hat{y}_{p}-y_{p} \in \mathbb{R}^{n_{e_{p}}}, e_{d}:=\hat{y}_{d}-y_{d} \in \mathbb{R}^{n_{e_{d}}}\left(n_{e_{u}}:=n_{u}\right.$ and $n_{e_{p}}=n_{e_{d}}:=n_{y}$ ) denote the network-induced errors on the feedback and the feedforward terms and the plant and the reference outputs, respectively. The functions $\hat{\mathbf{f}}_{f b}, \hat{\mathbf{f}}_{f f}, \hat{\mathbf{f}}_{p}, \hat{\mathbf{f}}_{d}$ represent the holding functions, i.e. the way the variables 
$\hat{u}_{f b}, \hat{u}_{f f}, \hat{y}_{p}, \hat{y}_{d}$ are generated between two successive transmission instants. In practice, it is common to use zero-orderhold devices, i.e. $\hat{\mathbf{f}}_{f b}, \hat{\mathbf{f}}_{f f}, \hat{\mathbf{f}}_{p}, \hat{\mathbf{f}}_{d}$ are equal to 0 . Other functions may also be implemented such as model-based algorithms as explained in [13], [14] for example. We let $\hat{\mathbf{f}}_{f b}, \hat{\mathbf{f}}_{f f}, \hat{\mathbf{f}}_{p}, \hat{\mathbf{f}}_{d}$ depend on $x_{p}, x_{c}$ and $x_{d}$ for the sake of generality to capture the cases where they depend on a part of these vector variables. The functions $\mathbf{h}_{f b}, \mathbf{h}_{f f}, \mathbf{h}_{p}, \mathbf{h}_{d}$ model the scheduling mechanism which governs the transmissions at each instant $t_{i}$ between the controller on the one hand and the plant and the reference system on the other hand. Following the terminology of [4], we refer to the equation below as the protocol

$$
\mathbf{e}\left(t_{i}^{+}\right)=\mathbf{h}\left(i, \mathbf{e}\left(t_{i}\right)\right)
$$

where $\mathbf{e}:=\left(e_{p}, e_{d}, e_{f b}, e_{f f}\right) \in \mathbb{R}^{n_{\mathbf{e}}}, n_{\mathbf{e}}:=n_{e_{p}}+n_{e_{d}}+2 n_{e_{u}}$, and $\mathbf{h}:=\left(\mathbf{h}_{p}, \mathbf{h}_{d}, \mathbf{h}_{f b}, \mathbf{h}_{f f}\right)$. Since the network is composed of $\ell$ nodes, we partition $\mathbf{e}$ as $\mathbf{e}=\left(\mathbf{e}_{1}, \ldots, \mathbf{e}_{\ell}\right)$ (after reordering, if necessary). The protocol (8) is such that at each transmission instant $t_{i}$, if node $j$ gets access to the network, the corresponding error $\mathbf{e}_{j}$ experiences a jump while the other components of $\mathbf{e}$ remain unchanged; usually $\mathbf{e}_{j}\left(t_{i}^{+}\right)=0$ but this is not needed in general. It has been shown in [4] that several common protocols can be modeled by (8). For the RR protocol which grants access to each node in a periodic fashion, the function $\mathbf{h}$ is given by

$$
\mathbf{h}(i, \mathbf{e})=(\mathbb{I}-\Delta(i)) \mathbf{e}
$$

where $\Delta(i)=\operatorname{diag}\left(\Delta_{1}(i), \ldots, \Delta_{\ell}(i)\right)$. For $k \in\{1, \ldots, \ell\}$ and $i \in \mathbb{Z}_{\geq 0}, \Delta_{k}(i):=\delta_{k}(i) \mathbb{I}_{n_{k}}$ where $\sum_{k \in\{1, \ldots, \ell\}} n_{k}=n_{\mathbf{e}}$ and $\delta_{k}(i)=1$ if $i=k+j l$ for $j \in \mathbb{Z}_{\geq 0}$ and $\delta_{k}(i)=0$ otherwise. The try-once-discard (TOD) protocol (introduced in [6]) gives access to the node where the norm of the local networkinduced error, $\left|\mathbf{e}_{j}\right|$ with $j \in\{1, \ldots, \ell\}$, is the largest. Therefore, we have

$$
\mathbf{h}(i, \mathbf{e})=(\mathbb{I}-\Psi(\mathbf{e})) \mathbf{e}
$$

where $\Psi(e):=\operatorname{diag}\left(\psi_{1}(\mathbf{e}) \mathbb{I}_{n_{1}}, \ldots, \psi_{\ell}(\mathbf{e}) \mathbb{I}_{n_{\ell}}\right)$ where $\psi_{j}(\mathbf{e})=$ 1 if $j=\min \left(\arg \max _{j^{\prime} \in\{1, \ldots, \ell\}}\left|\mathbf{e}_{j^{\prime}}\right|\right)$ and $\psi_{j}(\mathbf{e})=0$ otherwise. Model (8) also captures standard sampled-data systems (in which case there is no scheduling) by setting $\mathbf{h}$ to 0 .

Remark 1: When the output of the controller (5) is of the form $u_{f b}=\mathbf{g}_{c}\left(x_{c}, y_{p}, y_{d}\right)$ (instead of $u_{f b}=\mathbf{g}_{c}\left(x_{c}\right)$ ), the protocol (8) depends on $x_{p}, x_{d}$ and $x_{c}$ in general, i.e. $\mathbf{e}\left(t_{i}^{+}\right)=$ $\mathbf{h}\left(i, \mathbf{e}\left(t_{i}\right), x_{p}\left(t_{i}\right), x_{d}\left(t_{i}\right), x_{c}\left(t_{i}\right)\right)$. The model presented in the next section has to be modified accordingly in this case and the stability results of Section VI will apply; only the analysis of the protocol in Section VII needs to be revisited. It has to be noticed that there are situations in which the protocol (8) remains independent of $x_{p}, x_{d}, x_{c}$ when $u_{f b}=\mathbf{g}_{c}\left(x_{c}, y_{p}, y_{d}\right)$ (in which case the results of Section VII holds). This occurs for instance when the controller is directly connected to the plant actuators (as there is no error $e_{f b}$ ) or when there is no scheduling (as $\mathbf{h}=0$ ).

Our objective is to provide conditions on system (2)-(5) and on the network to guarantee the approximate convergence of the plant state $x_{p}$ towards the reference state $x_{d}$ in the presence of network-induced communication constraints.

\section{A Hybrid MOdel OF NCS}

Before presenting the hybrid model, we need to define new coordinates. As we are interested in the convergence of $x_{p}$ towards $x_{d}$, we introduce the tracking error $\xi:=x_{p}-x_{d} \in$ $\mathbb{R}^{n_{\xi}}\left(n_{\xi}=n_{x}\right)$. We also define the error $e:=\left(e_{\xi}, e_{f b}\right) \in \mathbb{R}^{n_{e}}$ where $e_{\xi}:=e_{p}-e_{d} \in \mathbb{R}^{n_{e_{\xi}}}, n_{e}:=n_{y}+n_{u}$ and $n_{e_{\xi}}:=n_{y}$. The idea is to show that the $\xi$ - and the $e$-systems satisfy some robust asymptotic stability properties with respect to the external perturbation vector $w:=\left(w_{p}, w_{d}, w_{c}\right) \in \mathbb{R}^{n_{w}}\left(n_{w}:=\right.$ $\left.n_{w_{p}}+n_{w_{d}}+n_{w_{c}}\right)$ and the network-induced errors $\left(e_{d}, e_{f f}\right)$ which are regarded as external disturbances similarly to [8]. This choice is motivated by the fact that $e_{d}$ and $e_{f f}$ typically depend on the reference system (3) and there is a priori no reason why they should satisfy some asymptotic stability properties even for very fast transmissions (recall that the MATI $\tau^{*}$ cannot be infinitely small as it needs to be such that $\tau^{*} \geq v>0$ ), contrary to $e$ as we will show in Section VI. For instance, when zero-order-hold devices are implemented, $\dot{e}_{d}=-\dot{y}_{d}$ and $\dot{e}_{f f}=-\dot{u}_{f f}$ so that the origin is not an equilibrium point of the systems in $e_{d}$ and $e_{f f}$ when $\dot{y}_{d} \neq 0$ and $\dot{u}_{f f} \neq 0$ (which is generally the case when tracking time-varying trajectories).

We model the overall NCS as a hybrid system using the formalism of [16], for which a jump describes a transmission. We use the coordinates $\left(\xi, x_{c}, x_{d}, e, e_{d}, e_{f f}, \kappa, \tau_{1}, \tau_{2}\right)$. The variable $\kappa \in \mathbb{Z}_{\geq 0}$ is a counter variable which keeps track of the number of transmissions. It is used to describe protocols such as the RR protocol where it plays the role of the discrete time $i$ in (9). The variables $\tau_{1}, \tau_{2} \in \mathbb{R}_{\geq 0}$ are clock variables: $\tau_{1}$ represents the time elapsed since the last transmission and $\tau_{2}$ models the 'continuous' time. The following model is derived:

$$
\left.\begin{array}{l}
\dot{\xi}=f_{\xi}\left(\tau_{2}, \xi, x_{c}, x_{d}, e, e_{d}, e_{f f}, w\right) \\
\dot{x}_{c}=f_{c}\left(\tau_{2}, \xi, x_{c}, x_{d}, e, e_{d}, w\right) \\
\dot{x}_{d}=f_{d}\left(\tau_{2}, x_{d}, w\right) \\
\dot{e}=g_{e}\left(\tau_{2}, \xi, x_{c}, x_{d}, e, e_{d}, e_{f f}, w\right) \\
\dot{e}_{d}=g_{d}\left(\tau_{2}, \xi, x_{c}, x_{d}, e, e_{d}, e_{f f}, w\right) \\
\dot{e}_{f f}=g_{f f}\left(\tau_{2}, \xi, x_{c}, x_{d}, e, e_{d}, e_{f f}, w\right) \\
\dot{\kappa}=0 \\
\dot{\tau}_{1}=1 \\
\dot{\tau}_{2}=1 \\
\xi^{+}=\xi \\
x_{c}^{+}=x_{c} \\
x_{d}^{+}=x_{d} \\
e^{+}=h_{e}\left(\kappa, e, e_{d}, e_{f f}\right) \\
e_{d}^{+}=h_{d}\left(\kappa, e, e_{d}, e_{f f}\right) \\
e_{f f}^{+}=h_{f f}\left(\kappa, e, e_{d}, e_{f f}\right) \\
\kappa^{+}=\kappa+1 \\
\tau_{1}^{+}=0 \\
\tau_{2}^{+}=\tau_{2}
\end{array}\right\} \tau_{1} \in\left[0, \tau^{*}\right]
$$

The functions $f_{\xi}, f_{c}, f_{d}, g_{e}, g_{d}, g_{f f}, h_{e}, h_{d}$ and $h_{f f}$ are obtained by direct calculations from the developments in Section III (the $\tau_{2}$-argument captures their dependency on $u_{f f}$ or $\dot{u}_{f f}$ ) and are assumed to be continuous. We similarly write $e_{p}^{+}=h_{p}\left(\kappa, e, e_{d}, e_{f f}\right)$ and $e_{f b}^{+}=h_{f b}\left(\kappa, e, e_{d}, e_{f f}\right)$ to model the jumps of the $e_{p}$-and the $e_{f b}$-systems at each transmission instant. 
For the sake of convenience, we introduce $q_{x}:=\left(\xi, x_{c}, x_{d}\right) \in$ $\mathcal{R}_{x}$ and $q_{e}:=\left(e, e_{d}, e_{f f}\right) \in \mathcal{R}_{e}$ to distinguish the physical variables from the errors induced by the network, where $\mathcal{R}_{x}:=$ $\mathbb{R}^{n_{\xi}+n_{x_{c}}+n_{x}}$ and $\mathcal{R}_{e}:=\mathbb{R}^{n_{e}+n_{e_{d}}+n_{e f f}}$. In that way, we can write

$$
\left.\begin{array}{l}
\dot{q}_{x}=f\left(\tau_{2}, q_{x}, q_{e}, w\right) \\
\dot{q}_{e}=g\left(\tau_{2}, q_{x}, q_{e}, w\right) \\
\dot{\kappa}=0 \\
\dot{\tau}_{1}=1 \\
\dot{\tau}_{2}=1 \\
q_{x}^{+}=q_{x} \\
q_{e}^{+}=h\left(\kappa, q_{e}\right) \\
\kappa^{+}=\kappa+1 \\
\tau_{1}^{+}=0 \\
\tau_{2}^{+}=\tau_{2}
\end{array}\right\} \tau_{1} \in[0
$$

\section{ASSUMPTIONS}

Inspired by [2], we present the assumptions we adopt which can be used as guidelines to design and implement the controller (4), (5) for the robust stabilisation of the desired trajectory.

The protocol has to be such that Assumption 1 holds.

Assumption 1: There exist a function $W: \mathbb{Z}_{\geq 0} \times \mathcal{R}_{e} \rightarrow \mathbb{R}_{\geq 0}$ that is locally Lipschitz in $q_{e}, \underline{\alpha}_{W}, \bar{\alpha}_{W} \in \mathcal{K}_{\infty}, \rho \in[0,1)$ and $\mu^{d}$, $\mu^{f f} \in \mathcal{K}_{\infty}$ such that for any $\left(\kappa, q_{e}\right) \in \mathbb{Z}_{\geq 0} \times \mathcal{R}_{e}$, it holds that

$$
\begin{aligned}
\underline{\alpha}_{W}(|e|) & \leq W\left(\kappa, q_{e}\right) \leq \bar{\alpha}_{W}\left(\left|q_{e}\right|\right), \\
W\left(\kappa+1, h\left(\kappa, q_{e}\right)\right) & \leq \rho W\left(\kappa, q_{e}\right)+\mu^{d}\left(\left|e_{d}\right|\right)+\mu^{f f}\left(\left|e_{f f}\right|\right) .
\end{aligned}
$$

The function $W$ is used to analyze the stability of the discrete-time dynamics of the $q_{e}$-system. We will see in Section VII that this system is strongly related to the scheduling protocol. It can be noted that $W$ is allowed to depend on the full vector $q_{e}$ but it needs to be lower bounded by a class- $\mathcal{K}_{\infty}$ function of $|e|$ according to (13). It is shown in Section VII that RR and TOD protocols admit a function $W$ which only depends on $e$. However, it is possible to envision protocols where $W$ does depend on the full vector $q_{e}$ (e.g. see Section VII-B). Contrary to similar conditions in [2]-[4], the second inequality in (13) holds with the additional perturbation terms $\mu^{d}$ and $\mu^{f f}$. This difference is due to the fact that Assumption 1 does not apply to the protocol (8) but to the $q_{e}$-system at jumps which, although related, are different dynamical systems. Indeed, the jumps of $q_{e}$ are governed by the vector field $h=$ $\left(h_{p}-h_{d}, h_{f b}, h_{f f}\right)$ while the protocol concerns the variable $\mathbf{e}$ whose jumps are dictated by $\mathbf{h}=\left(h_{p}, h_{d}, h_{f b}, h_{f f}\right)$. It can be noticed that analogous conditions to (13) are considered in [18] where input-to-state stable (ISS) protocols have been defined (except that here $e_{d}$ and $e_{f f}$ are parts of the overall state $q_{e}$, while in [18] there are exogenous disturbances). The constant $\rho$ in (13) often depends on the number of nodes $\ell$ of the network in such a way that large $\ell$ leads to large $\rho$, which tends to 1 as $\ell$ goes to infinity (as we will see in Section VII). This implies a smaller decrease of $W$ at each jump and therefore a smaller MATI bound according to the formula given in the following.

We assume that the following exponential growth condition on the $q_{e}$-dynamics between two transmission instants holds, which thus depends on the continuous-time dynamics of $y_{p}, y_{d}, u_{f b}, u_{f f}$ and on the choice of the holding functions.

Assumption 2: There exist $L \geq 0$, a continuous function $H$ : $\mathcal{R}_{x} \rightarrow \mathbb{R}_{\geq 0}$ and $\nu^{d}, \nu^{f f}, \nu^{w} \in \mathcal{K}_{\infty}$ such that for all $q_{x} \in \mathcal{R}_{x}$, $\kappa \in \mathbb{Z}_{\geq 0}, \tau_{2} \in \mathbb{R}_{\geq 0}, w \in \mathbb{R}^{n_{w}}$ and almost all $q_{e} \in \mathcal{R}_{e}$

$$
\begin{aligned}
&\left\langle\frac{\partial W\left(\kappa, q_{e}\right)}{\partial q_{e}}, g\left(\tau_{2}, q_{x}, q_{e}, w\right)\right\rangle \leq L W\left(\kappa, q_{e}\right)+H\left(q_{x}\right) \\
&+\nu^{d}\left(\left|e_{d}\right|\right)+\nu^{f f}\left(\left|e_{f f}\right|\right)+\nu^{w}(|w|)
\end{aligned}
$$

where $W$ comes from Assumption 1.

The controller (4), (5) needs to be designed so that the condition below is valid.

Assumption 3: There exist a locally Lipschitz function $V: \mathcal{R}_{x} \rightarrow \mathbb{R}_{\geq 0}, \underline{\alpha}_{V}, \bar{\alpha}_{V} \in \mathcal{K}_{\infty}, \varepsilon \in \mathbb{R}_{>0}, \gamma \in \mathbb{R}_{\geq 0}$ and $\sigma^{d}, \sigma^{f f}, \sigma^{w} \in \mathcal{K}_{\infty}$ such that for any $q_{x} \in \mathcal{R}_{x}$

$$
\underline{\alpha}_{V}(|\xi|) \leq V\left(q_{x}\right) \leq \bar{\alpha}_{V}\left(\left|q_{x}\right|\right)
$$

and for all $q_{e} \in \mathcal{R}_{e}, \tau_{2} \in \mathbb{R}_{\geq 0}, w \in \mathbb{R}^{n_{w}}$ and almost all $q_{x} \in$ $\mathcal{R}_{x}$

$$
\begin{gathered}
\left\langle\nabla V\left(q_{x}\right), f\left(\tau_{2}, q_{x}, q_{e}, w\right)\right\rangle \leq-\varepsilon V\left(q_{x}\right)-\varepsilon W^{2}\left(\kappa, q_{e}\right)-H^{2}\left(q_{x}\right) \\
\quad+\gamma^{2} W^{2}\left(\kappa, q_{e}\right)+\sigma^{d}\left(\left|e_{d}\right|\right)+\sigma^{f f}\left(\left|e_{f f}\right|\right)+\sigma^{w}(|w|)
\end{gathered}
$$

where $W$ and $H$ come from Assumptions 1-2.

The function $V$ may depend on the full vector $q_{x}$ but it needs to be lower bounded by a class- $\mathcal{K}_{\infty}$ function of the norm of $\xi$. This kind of Lyapunov functions is investigated in [19] in the context of the stability with respect to two measures for example. It relaxes standard requirements and it is sufficient to make statements about the convergence of the tracking error towards the origin. According to (14) and (15), the emulated controller does ensure an ISS-like property for the tracking error dynamics (i.e. the $\xi$-system) with $W, e_{d}, e_{f f}, w$ as inputs. Assumption 3 also implies that the $\xi$-system satisfies an $\mathcal{L}_{2}$-stability property from $\left(W, \sqrt{\sigma^{d}\left(\left|e_{d}\right|\right)}, \sqrt{\sigma^{f f}\left(\left|e_{f f}\right|\right)}, \sqrt{\sigma^{w}(|w|)}\right)$ to $H$. The constant $\varepsilon$ in (15) is usually taken sufficiently small. We will show how Assumptions 2 and 3 can be validated for particular (classes of) systems in Section IX.

The last condition is on the MATI. As in [2], we need to have a network which has a sufficiently high bandwidth so that the assumption stated below is satisfied.

Assumption 4: The MATI $\tau^{*}$ satisfies $\tau^{*}<\mathcal{T}(\rho, \gamma, L)$ where

$$
\mathcal{T}(\rho, \gamma, L):= \begin{cases}\frac{1}{L r} \arctan \left(\frac{r(1-\rho)}{2 \frac{\rho}{1+\rho}\left(\frac{\gamma}{L}-1\right)+1+\rho}\right) & \text { if } \gamma>L \\ \frac{1}{L} \frac{1-\rho}{1+\rho} & \text { if } \gamma=L \\ \frac{1}{L r} \operatorname{arctanh}\left(\frac{r(1-\rho)}{2 \frac{\rho}{1+\rho}\left(\frac{\gamma}{L}-1\right)+1+\rho}\right) & \text { if } \gamma<L\end{cases}
$$

with $r:=\sqrt{\left|(\gamma / L)^{2}-1\right|}, \rho \in[0,1)$ and $\gamma, L \geq 0$ come from Assumptions 1-3. 


\section{Main Results}

We are ready to state the main result. Its proof is based on the proof of Theorem 1 in [2] and requires some essential modifications to handle the effect of the network-induced errors $e_{d}, e_{f f}$ and external perturbations $w$.

Theorem 1: Consider system (12) and suppose Assumptions 1-4 hold. Then there exist $\beta \in \mathcal{K} \mathcal{L} \mathcal{L}, \delta^{d}, \delta^{f f}, \delta^{w} \in \mathcal{K}_{\infty}$ such that for any solution $\left(q_{x}, q_{e}, \kappa, \tau_{1}, \tau_{2}, w\right)$

$$
\begin{aligned}
& |(\xi(t, j), e(t, j))| \leq \beta\left(\left|\left(q_{x}(0,0), q_{e}(0,0)\right)\right|, t, j\right) \\
& \quad+\delta^{d}\left(\left\|e_{d}\right\|_{(t, j)}\right)+\delta^{f f}\left(\left\|e_{f f}\right\|_{(t, j)}\right)+\delta^{w}\left(\|w\|_{(t, j)}\right)
\end{aligned}
$$

for all $(t, j)$ in the domain of the solution. Moreover, $\delta^{d}(s)$ and $\delta^{f f}(s)$ can be written as $\left(1+\varphi\left(\tau^{*}\right)\right) \psi\left(v^{-1}\right) \delta(s)$ for $s \geq 0$ where $\delta, \psi \in \mathcal{K}_{\infty}$ and $\psi: \mathbb{R}_{\geq 0} \rightarrow \mathbb{R}_{>0}$.

Property (17) is obtained by constructing a hybrid Lyapunov function $U$ (see the proof of Theorem 1) which satisfies an ISSlike property on flows but not at jumps. Thus, we use the fact that $U$ flows for some time (at least $v$ seconds, see Section III-B) before jumping in order for the decreasing property of $U$ on flows to compensate, in some sense, the potential increase of $U$ at jumps.

Remark 2: The norms of the errors $\left\|e_{d}\right\|_{(t, j)},\left\|e_{f f}\right\|_{(t, j)}$ and the functions $\delta^{d}, \delta^{f f}$ in (17) depend on the MATI $\tau^{*}$. We may find upper bounds for $\left\|e_{d}\right\|_{(t, j)}$ and $\left\|e_{f f}\right\|_{(t, j)}$ on a case-by-case basis. For instance, when zero-order-hold devices are implemented and the RR protocol is selected, we can proceed like in (31) in [8] (where delays are taken into account but not scheduling). On the other hand, the functions $\delta^{d}, \delta^{f f}$ also depend on the minimum time $v$ between two jumps. We see that $\delta^{d}, \delta^{f f}$ go to infinity as $v$ tends to 0 . This fact is due to the stability analysis which requires to decrease for some time $v$ during flows in order to guarantee stability. On the other hand, the more transmissions, the smaller the norms of $e_{d}$ and $e_{f f}$, which would typically compensate the increase in the gains. That is the case in Section IX where all the gains are linear. The mean value theorem can then be used to upper bound the norms of $e_{d}$ and $e_{f f}$ by a constant that multiplies the inter-transmission interval (under mild regularity conditions on $y_{d}$ and $u_{f f}$ ) which would then compensate the constant $v$ coming for the gains. We think that a different analysis inspired by the small gain arguments used in [18] may help to avoid this issue. Nevertheless, our approach is justified by the fact that the proposed Lyapunov-based proof allows us to derive easily computable MATI bounds, which are typically less conservative than those derived using trajectory-based proofs, and that any real network has fixed minimum inter-transmission interval $v$.

Theorem 1 shows that $(\xi, e)$ tends to a ball centered at the origin and of radius ${ }^{1} \delta^{d}\left(\left\|e_{d}\right\|_{(t, j)}\right)+\delta^{f f}\left(\left\|e_{f f}\right\|_{(t, j)}\right)+$ $\delta^{w}\left(\|w\|_{(t, j)}\right)$ as $(t, j)$ grows. Thus, $\xi$ indeed converges to the origin up to some errors due to $w$, as expected, but also due

\footnotetext{
${ }^{1}$ If the maximal solutions to (12) are complete and if the limits superior of $\left\|e_{d}\right\|_{(t, j)},\left\|e_{f f}\right\|_{(t, j)},\left\|e_{w}\right\|_{(t, j)}$ are bounded as $t+j \rightarrow \infty$, a tighter upperbound of this radius is given by $\limsup \delta^{d}\left(\left|e_{d}(t, j)\right|\right)+\delta^{f f}\left(\left|e_{f f}(t, j)\right|\right)+$ $\delta^{w}(|w(t, j)|)$.
}

to $e_{f f}$ and $e_{d}$ which are induced by the network, similar to [8]. In practice, we want these errors to be sufficiently small and it might then be convenient to have some estimates of $\delta^{d}\left(\left\|e_{d}\right\|_{(t, j)}\right)$ and $\delta^{f f}\left(\left\|e_{f f}\right\|_{(t, j)}\right)$. While it may be possible to bound the norms of $e_{d}$ and $e_{f f}$ (see Remark 2), we know that the expressions for $\delta^{d}$ and $\delta^{f f}$ we can deduce from the proof of Theorem 1 are subject to some conservatism. Nevertheless, the result in Theorem 1 provides the following qualitative insights on how to reduce the impact of the network-induced errors $e_{f f}$ and $e_{d}$ on the tracking error:

- For $\delta^{f f}\left(\left\|e_{f f}\right\|_{(t, j)}\right)$ : first, when $u_{f f}$ can be directly implemented on the actuators, we have $e_{f f} \equiv 0$. When this is not possible, some previews of $u_{f f}$ might be considered as in [8] to reduce the error due to $e_{f f}$.

- For $\delta^{d}\left(\left\|e_{d}\right\|_{(t, j)}\right)$ : it can be shown that $\delta^{d}$ can be written as $\delta^{d}(s)=\alpha\left(\mu^{d}(s)+\nu^{d}(s)+\sigma^{d}(s)\right)$ for $s \geq 0$, where $\alpha$ is some class- $\mathcal{K}_{\infty}$ function (which depends on $V, W, \tau^{*}$ and $v)$ and $\mu^{d}, \nu^{d}, \sigma^{d}$ come from Assumptions 1-3. We show in Section VII that it is possible to set $\mu^{d}=0$ by selecting an appropriate protocol or by appropriately implementing the emulated controller.

In practice, we would like to make sure that the states $q_{x}=\left(\xi, x_{c}, x_{d}\right)$ and $q_{e}=\left(e, e_{d}, e_{f f}\right)$ remain bounded when the reference trajectory and the perturbation $w$ are bounded. This point is addressed in the proposition below.

Proposition 1: Consider system (12) and suppose the following holds.

(i) Assumptions 1-4 hold.

(ii) There exist some functions $N_{d}: \mathbb{R}^{n_{x+n_{e}}+n_{e f f}} \rightarrow \mathbb{R}_{\geq 0}$, $N_{c}: \mathbb{R}^{n_{x_{c}}} \rightarrow \mathbb{R}_{\geq 0}$ and $\gamma_{d}, \gamma_{c} \in \mathcal{K}_{\infty}$ such that for any solution $\left(q_{x}, q_{e}, \kappa, \tau_{1}, \tau_{2}, w\right)$

$$
\begin{aligned}
& \left|\left(x_{d}(t, j), e_{d}(t, j), e_{f f}(t, j)\right)\right| \\
& \quad \leq N_{d}\left(x_{d}(0,0), e_{d}(0,0), e_{f f}(0,0)\right)+\gamma_{d}\left(\|w\|_{(t, j)}\right)
\end{aligned}
$$

and

$$
\left|x_{c}(t, j)\right| \leq N_{c}\left(x_{c}(0,0)\right)+\gamma_{c}\left(\left\|\left(\xi, x_{d}, e, e_{d}, w\right)\right\|_{(t, j)}\right)
$$

for any $(t, j)$ in the domain of the solution. Then there exist a function $\bar{N}: \mathcal{R}_{x} \times \mathcal{R}_{e} \rightarrow \mathbb{R}_{\geq 0}$ and $\bar{\gamma} \in \mathcal{K}_{\infty}$ such that

$$
\left|\left(q_{x}(t, j), q_{e}(t, j)\right)\right| \leq \bar{N}\left(q_{x}(0,0), q_{e}(0,0)\right)+\bar{\gamma}\left(\|w\|_{(t, j)}\right)
$$

for all $(t, j)$ in the domain of the solution.

Item (i) of Proposition 1 implies that the assumptions of Theorem 1 hold so that (17) is ensured. Item (ii) of Proposition 1 gives conditions on the boundedness on the reference system (3) and the dynamic controller (5). Let us now illustrate how one could verify the conditions under item (ii) using reasonable assumptions for NCS. Consider for that purpose a solution $\left(q_{x}, q_{e}, \kappa, \tau_{1}, \tau_{2}, w\right)$ to $(12)$ and let $(t, j)$ be in the domain of the solution. The inequality (18) may be verified as follows. First, it may be shown that

$$
\left|x_{d}(t, j)\right| \leq N_{x_{d}}\left(x_{d}(0,0)\right)+\gamma_{x_{d}}\left(\|w\|_{(t, j)}\right)
$$


where $N_{x_{d}}: \mathbb{R}^{n_{x}} \rightarrow \mathbb{R}_{\geq 0}$ and $\gamma_{x_{d}} \in \mathcal{K}_{\infty}$, which is a reasonable assumption on the reference system when tracking bounded reference trajectories. For the $\left(e_{d}, e_{f f}\right)$-system, consider the case where zero-order-hold devices are implemented and the protocol is such that $\left|h_{d}\left(\kappa, e, e_{d}, e_{f f}\right)\right| \leq\left|e_{d}\right|$ and $\mid h_{f f}\left(\kappa, e, e_{d}\right.$, $\left.e_{f f}\right)|\leq| e_{f f} \mid$ (which is the case for any relevant protocol). When the norm of the feedforward term $u_{f f}$ is bounded by a constant $M_{f f} \geq 0$, we then derive that $\left|e_{f f}(t, j)\right| \leq 2 M_{f f}+$ $\left|e_{f f}(0,0)\right|$. Using (21) and the continuity of $\mathbf{g}_{p}$, we deduce that $\left|y_{d}(t, j)\right| \leq \widetilde{N}_{x_{d}}\left(x_{d}(0,0)\right)+\tilde{\gamma}_{x_{d}}\left(\|w\|_{(t, j)}\right)$ where $\widetilde{N}_{x_{d}}: \mathbb{R}^{n_{x}} \rightarrow$ $\mathbb{R}_{\geq 0}$ and $\tilde{\gamma}_{d} \in \mathcal{K}_{\infty}$. Hence $\left|e_{d}(t, j)\right| \leq\left|y_{d}(t, j)\right|+\left|\hat{y}_{d}(t, j)\right| \leq$ $2 \widetilde{\widetilde{N}}_{x_{d}}\left(x_{d}(0,0)\right)+2 \tilde{\gamma}_{x_{d}}\left(\|w\|_{(t, j)}\right)+\left|e_{d}(0,0)\right|$. In that way, (18) is satisfied with $N_{d}\left(x_{d}(0,0), e_{d}(0,0), e_{f f}(0,0)\right)=N_{x_{d}}\left(x_{d}(0\right.$, $0))+2 \widetilde{N}_{x_{d}}\left(x_{d}(0,0)\right)+\left|e_{d}(0,0)\right|+2 M_{f f}+\left|e_{f f}(0,0)\right|$ and $\gamma_{d}=$ $\gamma_{x_{d}}+2 \tilde{\gamma}_{x_{d}}$. Finally, the bounded-input-bounded-state property in (19) for the $x_{c}$-system may be studied using the Lyapunov function $V$ in Assumption 3 for instance.

\section{ON The Choice of the Protocol}

In this section, we give examples of protocols which ensure the satisfaction of Assumption 1 in Section V. We first show that this assumption is verified when the protocol (8) is Lyapunov UGAS under mild conditions. We then specialize this result for the RR protocol for which stronger properties are shown to hold. Finally, we propose a new dynamic TOD-like protocol.

\section{A. Lyapunov UGAS Protocols}

The stability of protocols has first been characterized in [4], and the notion of Lyapunov UGAS protocols has been introduced in [5].

Definition 1: The protocol (8) is said to be Lyapunov uniformly globally asymptotically stable (UGAS) if there exist $\mathbf{W}: \mathbb{Z}_{\geq 0} \times \mathbb{R}^{n_{\mathbf{e}}} \rightarrow \mathbb{R}_{\geq 0}, \underline{\boldsymbol{\alpha}}_{\mathbf{W}}, \overline{\boldsymbol{\alpha}}_{\mathbf{W}} \in \mathcal{K}_{\infty}$ and $\boldsymbol{\rho} \in[0,1)$ such that for all $\kappa \in \mathbb{Z}_{>0}$ and $\mathbf{e} \in \mathbb{R}^{n_{\mathbf{e}}}$ the following is satisfied:

$$
\begin{aligned}
\underline{\boldsymbol{\alpha}}_{\mathbf{W}}(|\mathbf{e}|) & \leq \mathbf{W}(\kappa, \mathbf{e}) \leq \overline{\boldsymbol{\alpha}}_{\mathbf{W}}(|\mathbf{e}|) \\
\mathbf{W}(\kappa+1, \mathbf{h}(\kappa, \mathbf{e})) & \leq \boldsymbol{\rho} \mathbf{W}(\kappa, \mathbf{e})
\end{aligned}
$$

recall that $\mathbf{e}=\left(e_{p}, e_{d}, e_{f b}, e_{f f}\right)$.

We are now ready to state the main result of this section.

Proposition 2: Consider the protocol (8) and suppose the following conditions hold.

(i) For any $j \in\left\{1, \ldots, n_{\mathbf{e}}\right\}$ and $\kappa \in \mathbb{Z}_{\geq 0},\left|\mathbf{h}_{j}(\kappa, \mathbf{e})\right| \leq\left|\mathbf{e}_{j}\right|$ with $\mathbf{h}=\left(\mathbf{h}_{1}, \ldots, \mathbf{h}_{n_{\mathbf{e}}}\right)$ where $\mathbf{h}$ is given in (8).

(ii) The protocol (8) is Lyapunov UGAS with a continuous function $\mathbf{W}: \mathbb{Z}_{\geq 0} \times \mathbb{R}^{n_{\mathbf{e}}} \rightarrow \mathbb{R}_{\geq 0}$ which is locally Lipschitz in e and satisfies for all $\kappa \in \mathbb{Z}_{>0}$ and almost all $\mathbf{e} \in \mathbb{R}^{n_{\mathbf{e}}},|\partial \mathbf{W}(\kappa, \mathbf{e}) / \partial \mathbf{e}| \leq M$, where $M \geq 0$.

Then Assumption 1 is verified with $W(\kappa, e)=\mathbf{W}\left(\kappa, e_{\xi}, 0, e_{f b}, 0\right)$, $\underline{\alpha}_{W}(s)=\underline{\boldsymbol{\alpha}}_{\mathbf{W}}(s), \quad \bar{\alpha}_{W}(s)=\overline{\boldsymbol{\alpha}}_{\mathbf{W}}(s), \quad \mu^{d}(s)=2 M(1+\boldsymbol{\rho}) s$, $\mu^{f f}(s)=M(1+\boldsymbol{\rho}) s$ for $s \geq 0$ and $\rho=\boldsymbol{\rho}$.

Note that item (i) in Proposition 2 simply states that the local errors do not increase at each transmission which is the case for all relevant protocols. The conditions of Proposition 2 are satisfied by the RR and the TOD protocol in view of Section IV in [4].
Since we are interested in a different stability property for the $e$-system at jumps than in [4], we can propose an alternative Lyapunov function to verify Assumption 1 for the RR protocol, based on Proposition 4 in [4], which ensures stronger properties and may lead to less conservative MATI bounds.

Proposition 3: Suppose the protocol (8) is the RR protocol as defined in (9), then Assumption 1 is satisfied with $W(\kappa, e)=$ $\sqrt{\sum_{i=\kappa}^{\infty}|\phi(i, \kappa, e)|^{2}}$, where $\phi(i, \kappa, e)$ is the solution to ${ }^{2} e^{+}=$ $\left(h_{p}\left(\kappa, e_{\xi}\right), h_{f b}\left(\kappa, e_{f b}\right)\right)$ at time $i$ starting at time $\kappa$ with initial condition $e, \underline{\alpha}_{W}(s)=s, \bar{\alpha}_{W}(s)=\sqrt{\ell} s, \mu^{d}(s)=\sqrt{\ell} s$ and $\mu^{f f}(s)=0$ for $s \geq 0$ and $\rho=\sqrt{(\ell-1) / \ell}$. Moreover, $\mu^{d}=0$ if and only if $h_{p}=h_{d}$.

Proposition 3 ensures the satisfaction of Assumption 1 with $\mu^{f f}=0$ which reduces the impact of the feedforward error $e_{f f}$ on the tracking error $\xi$. It also provides a necessary and sufficient condition to obtain $\mu^{d}=0$ in Assumption 1 which is interesting to reduce the impact of $e_{d}$ on the tracking error $\xi$ (see Section VI). That condition states that $\hat{y}_{p}$ and $\hat{y}_{d}$ must have the same dynamics at jumps which is the case when $y_{p}$ and $y_{d}$ are sent over the network via the same nodes for example. That also allows us to conclude that, even if $y_{d}$ (equivalently $y_{p}$ ) is directly available at the controller side, it may be advantageous to introduce the variable $\hat{y}_{d}$ (equivalently $\hat{y}_{p}$ ) to generate the control input instead of using $y_{d}$ (equivalently $y_{p}$ ), where $\hat{y}_{d}$ jumps as $\hat{y}_{p}$ does, otherwise $\mu^{d}$ will not be equal to 0 and it will introduce an additional error on the convergence of $(\xi, e)$. This is discussed in more detail in Section VIII and in the scope of an illustrative example in Section IX.

\section{B. The TOD-Tracking Protocol}

We now propose a new TOD-like protocol, that we call the TOD-tracking protocol. Consider the scenarios where each corresponding components of $y_{p}$ and $y_{d}$ are assigned to the same nodes. ${ }^{3}$ In that way, a subvector $\left(e, e_{f f}\right)_{j}$ of $\left(e, e_{f f}\right)$, $j \in\{1, \ldots, \ell\}$, can be associated to each of the $\ell$ nodes of the network. The idea is to grant access to the node where $\left|\left(e, e_{f f}\right)_{j}\right|$ is the biggest (and not $\left|\mathbf{e}_{j}\right|, j \in\{1, \ldots, \ell\}$, as in the classical TOD protocol, see the end of Section III-B). We define the function $\mathbf{h}$ in (8) as $\mathbf{h}(\kappa, \mathbf{e})=(\mathbb{I}-\Psi(\mathbf{e})) \mathbf{e}$ where $\Psi(\mathbf{e})=$ $\left(\delta_{1}(\mathbf{e}) \mathbb{I}_{n_{1}}, \ldots, \delta_{\ell}(\mathbf{e}) \mathbb{I}_{n_{\ell}}\right)$ where $n_{1}+\ldots+n_{\ell}=n_{\mathbf{e}}$ and

$$
\delta_{j}(\mathbf{e})= \begin{cases}1 & \text { if } j=\min \left(\arg \max _{j}\left|\left(e, e_{f f}\right)_{j}\right|\right) \\ 0 & \text { otherwise. }\end{cases}
$$

The lemma below shows that the TOD-tracking protocol satisfies Assumption 1. It directly follows from Proposition 5 in [4].

Proposition 4: Suppose the protocol (8) is the TODtracking protocol, then Assumption 1 is satisfied with $W\left(q_{e}\right)=$ $\left|\left(e, e_{f f}\right)\right|, \underline{\alpha}_{W}(s)=\bar{\alpha}_{W}(s)=s, \mu^{d}(s)=\mu^{f f}(s)=0$ for $s \geq$ 0 and $\rho=\sqrt{(\ell-1) / \ell}$.

The TOD-tracking protocol ensures Assumption 1 holds with $\mu^{d}=\mu^{f f}=0$, which is a priori not the case for the

\footnotetext{
${ }^{2}$ It has to be noted that $h_{p}$ (respectively $h_{d}$ ) only depends on $\kappa$ and $e_{p}$ (respectively $\kappa$ and $e_{d}$ ) for the RR protocol, see (9).

${ }^{3}$ The TOD-tracking protocol can also be used when the nodes which transmit $y_{p}$ (equivalently $y_{d}$ ) have access to $y_{d}$ (equivalently $y_{p}$ ). That is typically the case when $y_{d}$ is a given trajectory which can be implemented on smart nodes.
} 
TOD protocol according to Proposition 2. Thus, the TODtracking protocol may reduce the error of $(\xi, e)$, and hence improve the tracking performance in view of the discussion in Section VI. We will also see this in simulations for an example in Section IX.

Remark 3: Various variations of the TOD-tracking protocol can be deduced according to the network setup. For instance, when the control input is sent over the network as $u_{f b}+u_{f f}$, like in the example in Section IX-B, we can set the protocol to grant access to the node where $\left|\left(e_{\xi}, e_{f b}+e_{f f}\right)_{j}\right|$ is the largest (and not $\left|\left(e_{\xi}, e_{f b}, e_{f f}\right)_{j}\right|$ as above). We then take $W\left(q_{e}\right)=$ $\left|\left(e_{\xi}, e_{f b}+e_{f f}\right)\right|$. Assumption 1 is verified with the same functions $\underline{\alpha}_{W}, \bar{\alpha}_{W}, \mu^{d}, \mu^{f f}$ and constant $\rho$ as in Proposition 4, except that the lower bound in the first inequality of (13) depends on $\left|\left(e_{\xi}, e_{f b}+e_{f f}\right)\right|$ and not on $|e|$. In this case, (17) holds by replacing $e$ in the left hand-side by $\left(e_{\xi}, e_{f b}+e_{f f}\right)$.

\section{OBSERVER DESIGN}

In this section, we show how the results of Section VI can be used to emulate nonlinear observers for NCS. Consider the nonlinear system

$$
\dot{x}=\mathbf{f}(x, w), \quad y=\mathbf{g}(x)
$$

where $x \in \mathbb{R}^{n_{x}}$ is the state, $y \in \mathbb{R}^{n_{y}}$ the measured output, $w \in$ $\mathbb{R}^{n_{w}}$ is an external perturbation, $\mathbf{f}$ is continuous and $\mathbf{g}$ is continuously differentiable. We assume that we know how to design a full-order observer of the following form for system (25)

$$
\dot{\bar{x}}=\mathbf{f}(\bar{x}, 0)+\mathbf{k}(\bar{x}, y-\bar{y}), \quad \bar{y}=\mathbf{g}(\bar{x})
$$

where $\bar{x} \in \mathbb{R}^{n_{x}}$ is the estimate of $x, \bar{y} \in \mathbb{R}^{n_{y}}$ is the output of the observer and $\mathbf{k}$ is continuous. This problem can be seen as a tracking problem where we want $\bar{x}$ to converge towards $x$. We thus recover the formulation of Section III by taking

$$
\left\{\begin{array} { l } 
{ x _ { d } = x } \\
{ y _ { d } = y } \\
{ u _ { f f } = 0 } \\
{ w _ { d } = w }
\end{array} \quad \left\{\begin{array}{l}
x_{p}=\bar{x} \\
y_{p}=\bar{y} \\
u_{f b}=\mathbf{k}(\bar{x}, y-\bar{y}) \\
w_{p}=0
\end{array}\right.\right.
$$

$\mathbf{f}_{p}(x, u, w)=\mathbf{f}(x, w)+u$ and $\mathbf{g}_{p}=\mathbf{g}$. Notice that the innovation term of the observer $\mathbf{k}(\bar{x}, y-\bar{y})$ in (26) is interpreted as a feedback input to (26) which is directly sent to the observer.

We implement the observer (26) over a network, see Fig. 4. The output $y$ is sent over the communication channel via $\ell$ nodes. In [13], [14], the observer (26) is implemented as

$$
\dot{\bar{x}}=\mathbf{f}(\bar{x}, 0)+\mathbf{k}(\bar{x}, \hat{y}-\bar{y}) .
$$

Here, we do not necessarily make the emulated observer depend on its own output $\bar{y}$ but on some $\tilde{y}$ (which corresponds to $\hat{y}_{p}$ with the notation of Section III). In that way, the emulated observer is

$$
\dot{\bar{x}}=\mathbf{f}(\bar{x}, 0)+\mathbf{k}(\bar{x}, \hat{y}-\tilde{y}) .
$$

We will see that it is possible to ensure a stronger stability property than in [13] by appropriately selecting the dynamics of $\tilde{y}$. It has to be noticed that the same idea is proposed in [15] for the design of a class of high-gain observers. Compared to [15],

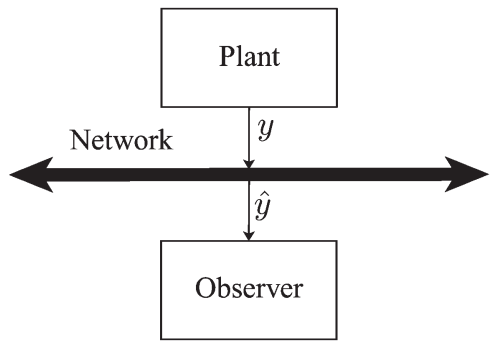

Fig. 4. Block diagram of the observer implementation over a network.

we treat a more general class of nonlinear observers and we propose a different stability analysis which leads to a different MATI bound formula. ${ }^{4}$

Noting that $e_{f f}=0$ since there is no feedforward term, we write the overall model using the coordinates $\left(\xi, x_{d}, e, e_{d}\right.$, $\left.\kappa, \tau_{1}\right)$ with $\xi=\bar{x}-x$, which we call the estimation error in this section, $x_{d}=x, e=e_{\xi}=e_{p}-e_{d}$ where $e_{p}=\tilde{y}-\bar{y}$ and $e_{d}=\hat{y}-y$

$$
\left.\begin{array}{l}
\dot{\xi}=f_{\xi}\left(\xi, x_{d}, e, w\right) \\
\dot{x}_{d}=f_{d}\left(x_{d}, w\right) \\
\dot{e}=g_{e}\left(\xi, x_{d}, e, w\right) \\
\dot{e}_{d}=g_{d}\left(\xi, x_{d}, e, w\right) \\
\dot{\kappa}=0 \\
\dot{\tau}_{1}=1 \\
\xi^{+}=\xi \\
x_{d}^{+}=x_{d} \\
e^{+}=h_{e}\left(\kappa, e, e_{d}\right) \\
e_{d}^{+}=h_{d}\left(\kappa, e, e_{d}\right) \\
\kappa^{+}=\kappa+1 \\
\tau_{1}^{+}=0
\end{array}\right\} \tau_{1} \in\left[0, \tau^{*}\right]
$$

with

$$
\begin{aligned}
f_{\xi}\left(\xi, x_{d}, e, w\right):= & \mathbf{f}\left(\xi+x_{d}, 0\right)-\mathbf{f}\left(x_{d}, w\right) \\
& +\mathbf{k}\left(\xi+x_{d}, \mathbf{g}\left(x_{d}\right)-\mathbf{g}\left(x_{d}+\xi\right)-e\right) \\
f_{d}\left(x_{d}, w\right):= & \mathbf{f}(x, w)=\mathbf{f}\left(x_{d}, w\right) \\
g_{e}\left(\xi, x_{d}, e, w\right):= & \hat{\mathbf{f}}_{p}\left(\xi, x_{d}, e, w\right)-\hat{\mathbf{f}}_{d}\left(\xi, x_{d}, e, w\right) \\
& +\frac{\partial \mathbf{g}}{\partial x}\left(x_{d}\right) \mathbf{f}\left(x_{d}, w\right)-\frac{\partial \mathbf{g}}{\partial \bar{x}}\left(x_{d}+\xi\right) \\
& \times\left(\mathbf{f}\left(\xi+x_{d}, 0\right)\right. \\
& \left.\quad+\mathbf{k}\left(\xi+x_{d}, \mathbf{g}\left(x_{d}\right)-\mathbf{g}\left(x_{d}+\xi\right)-e\right)\right) \\
g_{d}\left(\xi, x_{d}, e, w\right):= & \hat{\mathbf{f}}_{d}\left(\xi, x_{d}, e, w\right)-\frac{\partial \mathbf{g}}{\partial x}\left(x_{d}\right) \mathbf{f}\left(x_{d}, w\right)
\end{aligned}
$$

where $\hat{\mathbf{f}}_{p}$ and $\hat{\mathbf{f}}_{d}$ are defined by the holding functions. We do not need to introduce the variable $\tau_{2}$ as in (11) because there is no feedforward term here. Since the problem can be modeled as in Section IV, we can directly apply Theorem 1 to conclude about the convergence of the estimation error $\xi$ under the required conditions.

On the other hand, it may be possible to select the dynamics of $\hat{y}_{p}=\tilde{y}$ so that (17) holds with $\delta^{d}=0$, i.e. the estimation error converges to a smaller neighborhood of the origin. To see this, consider the case where zero-order-hold devices are

\footnotetext{
${ }^{4}$ It is hard to say that the bound in Corollary 1 is less or more conservative than the bounds in [15] or [13] in general because they are based on a different set of assumptions and do not depend on the same constants.
} 
TABLE I

CONSTANTS USED IN SECTION VIII

\begin{tabular}{cccc}
\hline & RR & TOD-tracking & Sampled-data \\
\hline$\rho$ & $\sqrt{\frac{\ell-1}{\ell}}$ & $\sqrt{\frac{\ell-1}{\ell}}$ & 0 \\
$M$ & $\sqrt{l}$ & 1 & 1 \\
$L$ & $\tilde{L} \sqrt{\ell}$ & $\tilde{L}$ & $\tilde{L}$ \\
\hline
\end{tabular}

used (i.e. $\hat{\mathbf{f}}_{p}=\hat{\mathbf{f}}_{d}=0$ in (31)) and the protocol is either the RR, the TOD-tracking protocol ${ }^{5}$ or all data are transmitted at each transmission instant as in the context of sampled-data systems. The variable $\tilde{y}$ is held constant between two transmissions and jumps as $\hat{y}$ does, i.e., when $\hat{y}^{i}$ for $i \in\{1, \ldots, \ell\}$ is updated so is $\tilde{y}$. Denoting $\tilde{y}=\left(\tilde{y}_{1}, \ldots, \tilde{y}_{n_{y}}\right), \hat{y}=\left(\hat{y}_{1}, \ldots, \hat{y}_{n_{y}}\right)$ and $y=\left(y_{1}, \ldots, y_{n_{y}}\right)$, the dynamics of $\tilde{y}$ is given by

$$
\begin{aligned}
\dot{\tilde{y}} & =0 \\
\tilde{y}_{j}^{+} & =\left\{\begin{array}{lll}
\bar{y}_{j} & \text { if } \hat{y}_{j}^{+}=y_{j} & \text { when } \tau_{1} \in\left[0, \tau^{*}\right] \\
\tilde{y}_{j} & \text { otherwise } & \text { when } \tau_{1} \in\left[v, \tau^{*}\right] .
\end{array}\right.
\end{aligned}
$$

Note that, in that case, the system can be modeled as in (30) with a jump map for the $e$-system which is continuous. In that way, Assumption 1 is valid with $\mu^{d}=0$ according to Propositions 3-4, respectively, for the RR and the TODtracking protocols. We make the following assumption which is satisfied by the observers in [20]-[22] for instance when using zero-order-hold devices.

Assumption 5: There exist $\tilde{L} \geq 0$, a continuous function $\tilde{H}$ : $\mathbb{R}^{n_{\xi}} \rightarrow \mathbb{R}_{>0}$ and $\tilde{\nu}^{w} \in \mathcal{K}_{\infty}$ such that for all $\xi \in \mathbb{R}^{n_{\xi}}, x_{d} \in \mathbb{R}^{n_{x}}$, $e \in \mathbb{R}^{n_{e}}, e_{d} \in \mathbb{R}^{n_{e_{d}}}$ and $w \in \mathbb{R}^{n_{w}}$, it holds that

$$
\left|g_{e}\left(\xi, x_{d}, e, w\right)\right| \leq \tilde{L}|e|+\tilde{H}(\xi)+\tilde{\nu}^{w}(|w|) .
$$

We take the function $W$ to be as in Proposition 3 for the RR protocol and we choose $W(e)=|e|$ for the TOD-tracking protocol (note that $e_{f f}=0$ here) and for the sampled-data case. Thus, by combining Assumption 5 with the fact that for the considered protocols, for all $\kappa \in \mathbb{Z}_{\geq 0}$ and almost all $e \in \mathbb{R}^{n_{e}}$ it holds that

$$
\left|\frac{\partial W(\kappa, e)}{\partial e}\right| \leq M
$$

where $M \geq 0$ is given in Table I. Assumption 2 is then satisfied with $L=M \tilde{L}, H=M \tilde{H}, \nu^{d}=0$, and $\nu^{w}=M \tilde{\nu}^{w}$.

Finally, the observer needs to be designed such that Assumption 3 is satisfied with $\sigma^{d}=0$. This is justified by the definition of the vector fields of system in (30) which can be written independently of $e_{d}$, see (31) (recall that $\hat{\mathbf{f}}_{p}=\hat{\mathbf{f}}_{p}=0$ here). In that way, property (17) holds with $\delta^{d}=\delta^{f f}=0$ for system (30) as stated below.

Colorary 1: Consider system (30) with either the RR or the TOD-tracking protocol or in the sampled-data case. Suppose Assumption 5 is satisfied and Assumption 3 holds with $\sigma^{d}=0$.

\footnotetext{
${ }^{5}$ When the TOD-tracking protocol is implemented, we need the sensor nodes to have access to $y_{p}$ (and thus $e_{p}$ ), i.e. they need to have sufficient computational capacities to run a copy of the observer; a similar implementation is described in more detail in Remark 2 in [13].
}

If the MATI $\tau^{*}$ is strictly less than $\mathcal{T}(\rho, \gamma, L)$ in (16) where $\gamma$ comes from Assumption 3 and $L$ and $\rho$ are given in Table I depending on the adopted protocol, then there exist $\beta \in \mathcal{K} \mathcal{L} \mathcal{L}$, $\delta^{w} \in \mathcal{K}_{\infty}$ such that for any solution $\left(\xi, x_{d}, e, e_{d}, \kappa, \tau_{1}, w\right)$

$$
|(\xi(t, j), e(t, j))| \leq \beta\left(\left|\left(q_{x}(0,0), e(0,0)\right)\right|, t, j\right)+\delta^{w}\left(\|w\|_{(t, j)}\right)
$$

for all $(t, j)$ in the domain of the solution.

Compared to [13], we do not require the plant (25) to be stable and we ensure the asymptotic convergence of the estimation error towards the origin in the absence of perturbations $w$ (as opposed to a practical stability property in [13]) when the observer (26) is emulated using zero-order-hold devices. Furthermore, a new MATI bound is given in Corollary 1.

\section{EXAMPLES}

We demonstrate how the results of Section VI can be used for the tracking control of stabilizable linear systems in Section IXA. We then consider an example concerning a nonlinear singlelink robot arm in Section IX-B.

\section{A. Linear Systems}

Consider the linear plant $\dot{x}_{p}=A x_{p}+B u+F w_{p}$ where $A, B, C$ are real matrices of appropriate dimensions, the pair $(A, B)$ is stabilizable and the state is measured $\left(y_{p}=x_{p}\right.$ in (2)). The feedforward term $u_{f f}$ verifies $\dot{x}_{d}=A x_{d}+B u_{f f}$, where $x_{d}$ is also measured $\left(y_{d}=x_{d}\right.$ in (3)). We assume that $x_{d}(t)$ is twice continuously differentiable so that $u_{f f}(t)$ is continuously differentiable. The controller is designed as $u=u_{f b}+u_{f f}$ with $u_{f b}=-K\left(x_{p}-x_{d}\right)$ where $K$ is such that $A-B K$ is Hurwitz. It ensures the asymptotic convergence of $x_{p}$ towards the reference trajectory $x_{d}$ up to an error due to $w_{p}$. We implement the controller over a network composed of $\ell$ nodes, as described in Section III, using zero-order-hold devices. The scheduling protocol is selected to be the RR protocol; noting that similar results can be derived for the TOD(-tracking) protocols. We write the problem using the model in (11). We obtain

$$
\begin{aligned}
f_{\xi}\left(\xi, e, e_{f f}, w\right) & =(A-B K) \xi+B\left(\Lambda e+e_{f f}\right)+F w_{p} \\
f_{d}\left(\tau_{2}, x_{d}\right) & =A x_{d}+B u_{f f} \\
g_{e}\left(\xi, e, e_{f f}, w\right) & =\left(-(A-B K) \xi-B\left(\Lambda e+e_{f f}\right)-F w_{p}, 0\right) \\
g_{d}\left(\tau_{2}, x_{d}\right) & =-A x_{d}-B u_{f f}
\end{aligned}
$$

where $\Lambda=[-K \mathbb{I}]$ and recall that $\tau_{2}$ reflects time-dependencies in the right-hand side due to $u_{f f}$. We concentrate on the case where the plant state $x_{p}$ and the reference trajectory $x_{d}$ are transmitted to the controller via distinct nodes. In that case, we assume that $u_{f f}$ is sent from the reference system to the actuators via the network, as depicted in Fig. 3. The same approach can be applied for the other cases described in Section III-B.

Since $A-B K$ is Hurwitz, the $\xi$-system is $\mathcal{L}_{2}$-gain stable from $\left(e, e_{f f}, w_{p}\right)$ to $(A-B K) \xi$ with gain $\gamma \geq 0$. The result below follows from Theorem 1. Its proof is omitted; it consists in verifying that the required conditions of Theorem 1 holds for this particular linear case. 
Proposition 5: Consider system (11) with (36) and suppose $\tau^{*}$ satisfies Assumption 4 with $\rho=\sqrt{(\ell-1) / \ell}, L=\sqrt{\ell}|B \Lambda|$ and $\gamma$ is defined above. Then property (17) holds.

\section{B. Single-Link Robot Arm}

We consider a single-link robot arm whose dynamics can be written as

$$
\dot{x}_{1}=x_{2}, \quad \dot{x}_{2}=-a \sin \left(x_{1}\right)+b u
$$

where $x_{1}$ is the angle, $x_{2}$ is the rotational velocity which are both measured, $u$ is the input torque and $a, b>0$ are fixed parameters. The system (37) has to track the reference system

$$
\dot{x}_{1, d}=x_{2, d}, \quad \dot{x}_{2, d}=-a \sin \left(x_{1, d}\right)+b u_{f f}
$$

where $x_{1, d}$ and $x_{2, d}$ are measured and $u_{f f}(t)=10 \sin (50 t)$. When there is no communication constraint, the asymptotic convergence of $\left(x_{1}, x_{2}\right)$ towards $\left(x_{1, d}, x_{2, d}\right)$ is ensured using the control input $u=u_{f b}+u_{f f}$ where $u_{f b}=b^{-1}\left(a\left(\sin \left(x_{1}\right)-\right.\right.$ $\left.\left.\sin \left(x_{1, d}\right)\right)-\left(x_{1}-x_{1, d}\right)-\left(x_{2}-x_{2, d}\right)\right)$. We consider the case where the controller is implemented using zero-order-hold devices and communicates with system (37) via a network composed of 3 nodes for $x_{1}, x_{2}$ and $u$, respectively $(\ell=3)$. Thus, we assume that ${ }^{6} x_{1, d}, x_{2, d}, u_{f f}$ are directly available to the controller as in Fig. 2. The transmission sequence $\left\{t_{i}\right\}_{i \in \mathbb{Z}_{>0}}$ is such that $t_{i}-t_{i-1}=\tau^{*}(=v)$ for $i \in \mathbb{Z}_{>0}$, where $\tau^{*}$ will be specified later. The emulated feedback controller is

$$
u_{f b}=b^{-1}\left(a\left(\sin \left(\hat{x}_{1}\right)-\sin \left(\hat{x}_{1, d}\right)\right)-\left(\hat{x}_{1}-\hat{x}_{1, d}\right)-\left(\hat{x}_{2}-\hat{x}_{2, d}\right)\right)
$$

where $\hat{x}_{1, d}$ and $\hat{x}_{2, d}$ are held constant between transmissions and jump as $\hat{x}_{1}$ and $\hat{x}_{2}$ do. In that way, the emulated feedback term (39) does not depend on $x_{1, d}$ and $x_{2, d}$ although these variables are continuously known by the controller. We will see that this choice may be advantageous in order to reduce the impact of the errors $e_{d}$ and $e_{f f}$ on the convergence of the tracking error.

In the sequel, we study three different protocols: the RR, the TOD and the TOD-tracking. We write the system in the form of (11) with: $f_{\xi}\left(q_{x}, q_{e}\right)=\left(\xi_{2},-a\left(\sin \left(\xi_{1}+x_{1, d}\right)-\sin \left(x_{1, d}\right)-\right.\right.$ $\left.\sin \left(\xi_{1}+x_{1, d}+e_{1, \xi}+e_{1, d}\right)+\sin \left(x_{1, d}+e_{1, d}\right)\right)-\left(\xi_{1}+e_{1, \xi}\right)-$ $\left.\left(\xi_{2}+e_{2, \xi}\right)+b e_{f b}+b e_{f f}\right), f_{d}\left(\tau_{2}, x_{d}\right)=\left(x_{2, d},-a \sin \left(x_{1, d}\right)+\right.$ $\left.b u_{f f}\right), g_{e}\left(q_{x}, q_{e}\right)=-\left(f_{\xi}\left(q_{x}, q_{e}\right), 0\right), g_{d}\left(\tau_{2}, q_{x}\right)=-f_{d}\left(\tau_{2}, x_{d}\right)$ and $g_{f f}\left(\tau_{2}\right)=-\dot{u}_{f f}$. We consider the function $W$ in Proposition 3 for the RR protocol, $W(e)=|e|$ for the TOD protocol and $W\left(q_{e}\right)=\left|\left(e_{\xi}, e_{f b}+e_{f f}\right)\right|$ for the TOD-tracking protocol (see Remark 3). In that way, Assumption 1 is valid, see Section VII. On the other hand, we have that $\left|g_{e}\left(q_{x}, q_{e}\right)\right| \leq$ $\left|\xi_{2}\right|+\left|\xi_{1}+\xi_{2}\right|+D|e|+2 a\left|e_{d}\right|+b\left|e_{f f}\right|$, where $D:=\sqrt{3} \max$ $\{1+a, b\}$. The considered functions $W$ are such that: $\underline{\alpha}_{W}(s)=s$ for $s \geq 0$ and $\left|\partial W\left(\kappa, q_{e}\right) / \partial q_{e}\right| \leq M$ for almost all $q_{e}$ and all $\kappa$ with $M=\sqrt{\ell}$ for the RR protocol (see Example 3 in [4]) and $M=1$ for the TOD and the

\footnotetext{
${ }^{6} \mathrm{We}$ make this assumption in order to be able to consider the TOD-tracking protocol (see Section VII).
}

TABLE II

MATI BOUNDS IN SECTION IX-B

\begin{tabular}{lccc}
\hline & RR & TOD & TOD-tracking \\
\hline Assumption 4 & 0.0050 & 0.0061 & 0.0061 \\
Simulations & 0.150 & 0.170 & 0.170 \\
\hline
\end{tabular}

TOD-tracking protocol. As a consequence, $\mid\left\langle\left(\partial W\left(\kappa, q_{e}\right) \mid\right.\right.$ $\left.\left.\partial q_{e}\right), g\left(\tau_{2}, q_{x}, q_{e}, w\right)\right\rangle \mid \leq M\left(D W(\kappa, e)+\left|\xi_{2}\right|+\left|\xi_{1}+\xi_{2}\right|+2 a\right.$ $\left.\left|e_{d}\right|+b\left|e_{f f}\right|\right)$ for almost all $q_{e}$ and all $q_{x}, w, \tau_{2}, \kappa$, where $g=$ $\left(g_{e}, g_{d}\right)$. Hence, Assumption 2 is verified with $L=M D$, $H\left(q_{x}\right)=M\left(\left|\xi_{2}\right|+\left|\xi_{1}+\xi_{2}\right|\right), \nu^{d}(s)=2 M a s$ and $\nu^{f f}(s)=$ $M b s$ for $s \geq 0$. We now show that Assumption 3 holds with $V(\xi)=\alpha \xi_{1}^{2}+\beta \xi_{1} \xi_{2}+\delta \xi_{2}^{2}$ where $\alpha, \beta, \delta$ will be chosen such that (14) holds. Writing $a\left(\sin \left(\xi_{1}+x_{1, d}\right)-\sin \left(\xi_{1}+x_{1, d}+e_{1, \xi}+\right.\right.$ $\left.\left.e_{1, d}\right)\right)=\bar{a}\left(e_{1, \xi}+e_{1, d}\right)$ and $a\left(\sin \left(x_{1, d}\right)-\sin \left(x_{1, d}+e_{1, d}\right)\right)=$ $\tilde{a} e_{1, d}$ with varying parameters $\bar{a}, \tilde{a}$ in $[-a, a]$, we have that $\left\langle\nabla V(\xi), f_{\xi}\left(q_{x}, q_{e}\right)\right\rangle \leq-\beta \xi_{1}^{2}-(2 \delta-\beta) \xi_{2}^{2}+(2 \alpha-2 \delta-\beta) \xi_{1} \xi_{2}+$ $\left(2 \delta \xi_{2}+\beta \xi_{1}\right)\left(\Upsilon e+(-\bar{a}+\tilde{a}) e_{1, d}+b e_{f f}\right)$ where $\Upsilon:=[-\bar{a}-1$ $-1 b]$. Applying twice the fact that $x y \leq(\eta / 2) x^{2}+(1 / 2 \eta) y^{2}$ for $x, y \in \mathbb{R}_{\geq 0}$ and $\eta>0$, we obtain $\left\langle\nabla V(\xi), f_{\xi}\left(q_{x}, q_{e}\right)\right\rangle \leq-\beta$ $\xi_{1}^{2}-(2 \delta-\beta) \xi_{2}^{2}+(2 \alpha-2 \delta-\beta) \xi_{1} \xi_{2}+(1 / 2)\left(\eta^{-1}+\tilde{\eta}^{-1}\right)\left(2 \delta \xi_{2}+\right.$ $\left.\beta \xi_{1}\right)^{2}+(1 / 2) \eta D^{2}|e|^{2}+(1 / 2) \tilde{\eta}\left((-\bar{a}+\tilde{a}) e_{1, d}+b e_{f f}\right)^{2}$ where $\eta, \tilde{\eta}>0$ and $D$ has been defined above. We use that $\mid-\bar{a}+$ $\tilde{a} \mid \leq 2 a$ and $(x+y)^{2} \leq 2 x^{2}+2 y^{2}$ to obtain $\left\langle\nabla V(\xi), f_{\xi}\left(q_{x}\right.\right.$, $\left.\left.q_{e}\right)\right\rangle \leq-\beta \xi_{1}^{2}-(2 \delta-\beta) \xi_{2}^{2}+\quad(2 \alpha-2 \delta-\beta) \xi_{1} \xi_{2}+(1 / 2)\left(\eta^{-1}+\right.$ $\left.\tilde{\eta}^{-1}\right)\left(2 \delta \xi_{2}+\beta \xi_{1}\right)^{2}+\quad(1 / 2) \eta D^{2}|e|^{2}+\tilde{\eta}\left(4 a^{2}\left|e_{d}\right|^{2}+b^{2}\left|e_{f f}\right|^{2}\right)$. Therefore, if we ensure that (14) holds and

$$
\begin{array}{r}
-\varepsilon|\xi|^{2}-H^{2}\left(q_{x}\right) \geq-\beta \xi_{1}^{2}-(2 \delta-\beta) \xi_{2}^{2}+(2 \alpha-2 \delta-\beta) \xi_{1} \xi_{2} \\
+\frac{1}{2}\left(\eta^{-1}+\tilde{\eta}^{-1}\right)\left(2 \delta \xi_{2}+\beta \xi_{1}\right)^{2}
\end{array}
$$

with $\varepsilon>0$, then Assumption 2 is verified with $\gamma=$ $\sqrt{(1 / 2) \eta D^{2}+\varepsilon}, \sigma^{d}(s)=4 \tilde{\eta} a^{2} s^{2}$ and $\sigma^{f f}(s)=\tilde{\eta} b^{2} s^{2}$ for $s \geq 0$. Note that Assumption 2 holds when $\alpha=\beta=\delta$ and by taking $\alpha, \eta$ and $\tilde{\eta}$ sufficiently large and $\varepsilon$ sufficiently small. Nonetheless, such a choice may lead to a large $\gamma$ which may then give us conservative MATI bounds (as the bound in (16) increases as $\gamma$ increases). Thus, we have computed $\alpha, \beta, \delta, \eta$ by minimizing $\gamma=\sqrt{(1 / 2) \eta D^{2}+\varepsilon}$ under the conditions that (14) and (40) hold using the Matlab optimization toolbox taking $a=9.81 \cdot 0.5$ and $b=2$. We have obtained $\alpha=3.05, \beta=$ $1.05, \delta=5.05, \eta=10.11$ and $\varepsilon=0.0001$. The MATI bounds are summarized and compared to the bounds estimated via simulations in Table II.It has to be emphasized that our method strongly relies on the choice of the Lyapunov functions $V$ and $W$ and that other functions may lead to larger bounds. We notice that the bounds for the TOD and the TOD-tracking protocol are the same according to Assumption 4 and in simulations. Interest in the TOD-tracking is justified by the fact that it may reduce the impact of the errors $e_{d}$ and $e_{f f}$ on the tracking error as discussed below Proposition 4 and illustrated by Fig. 5. On the other hand, we see in Fig. 6 that the convergence error is of the same order of magnitude when using the TOD-tracking and the RR protocol; the advantage of the TOD-tracking is that we can consider larger transmission intervals (see Table II). Finally, we have compared the obtained tracking errors for the cases where the emulated feedback controller (39) uses either 

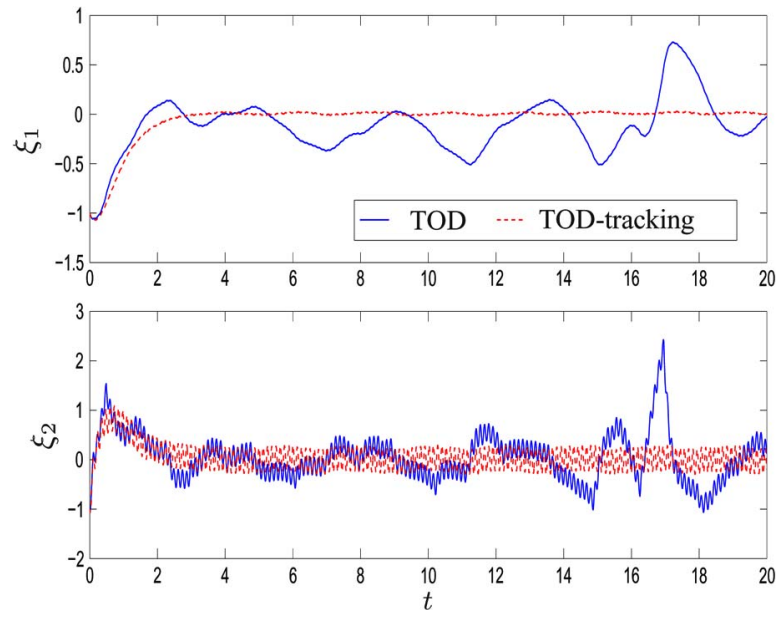

Fig. 5. Tracking error for MATI $\tau^{*}=0.005$
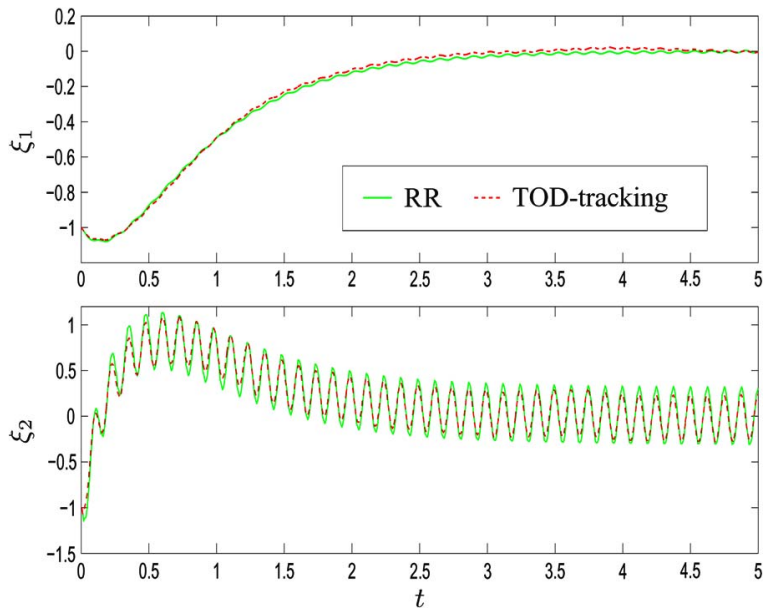

Fig. 6. Tracking error for MATI $\tau^{*}=0.005$.
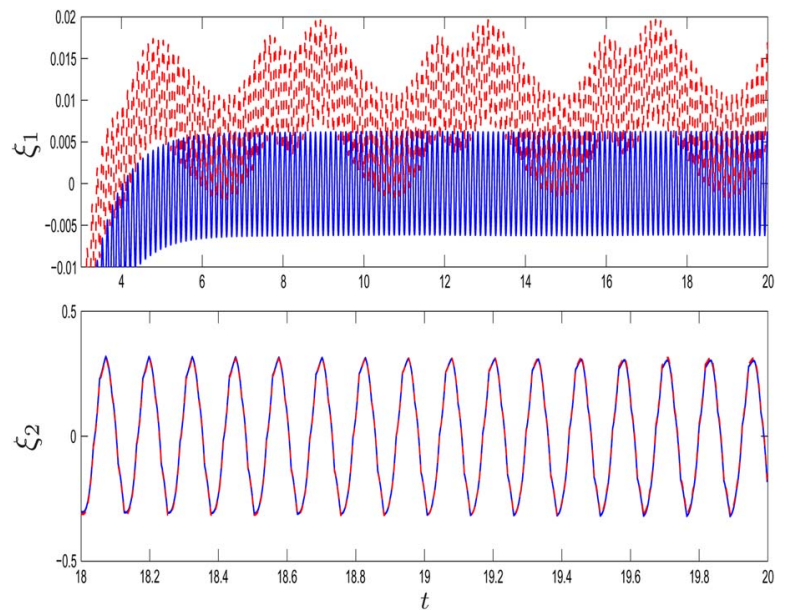

Fig. 7. Tracking error for MATI $\tau^{*}=0.005$ and the RR protocol when the controller uses $\left(\hat{x}_{1, d}, \hat{x}_{2, d}\right)$ (solid lines) or $\left(x_{1, d}, x_{2, d}\right)$ (dashed lines).

the variables $\left(\hat{x}_{1, d}, \hat{x}_{2, d}\right)$ or $\left(x_{1, d}, x_{2, d}\right)$ in (39), see Fig. 7. We see that, for the RR protocol, $\xi_{1}:=x_{1}-x_{1, d}$ converges to a smaller neighborhood of the origin when the controller uses $\left(\hat{x}_{1, d}, \hat{x}_{2, d}\right)$ instead of $\left(x_{1, d}, x_{2, d}\right)$, while no major difference is seen for $\xi_{2}:=x_{2}-x_{2, d}$.

\section{COnClusion}

We have presented a Lyapunov-based emulation approach for the tracking control of time-varying trajectories for nonlinear NCS. To handle the specific features of tracking control for NCS, we have proposed a new hybrid model. We have presented sufficient conditions under which an approximate tracking control objective is achieved. In addition, we have explained how the controller can be implemented and how the protocol can be set up in order to reduce the impact of some of the network-induced errors on the tracking error. Finally, it has been shown that these results on tracking control can be directly employed to obtain new results for the observer design problem for NCS as well. We believe that the results of this paper can be extended in various directions. In particular, tracking control in NCS subject to small transmission delays can be addressed by first appropriately modifying the model of Section IV and then adapting the Lyapunov-based stability analysis given in [3].

\section{APPENDIX}

Proof of Theorem 1: The proof is organised as follows. First, a hybrid Lyapunov function $U$ is designed. Second, we study the derivative of $U$ along the solutions to (11) on flows (when $\tau_{1} \in\left[0, \tau^{*}\right]$ ) and its dynamics at jumps (when $\tau_{1} \in\left[v, \tau^{*}\right]$ ). Third, we obtain (17) by applying standard comparison principles together with the fact there exists a minimum amount of time $v$ between two jumps. Finally, we prove the last part of Theorem 1 about the functions $\delta^{d}, \delta^{f f}$.

We focus on the case where $\rho \in(0,1)$; when $\rho=0$ similar arguments as in [24] are used. The constant $\mathcal{T}(\rho, \gamma, L)$ in (16) corresponds to the time it takes for the solution to $\dot{\psi}=-2 L \psi-$ $\gamma\left(\psi^{2}+1\right)$ to decrease from the initial condition $\psi(0)=1 / \rho$ to $\psi(\mathcal{T}(\rho, \gamma, L))=\rho$ (see Lemma 2 in [2]). We now define the following differential system

$$
\dot{\phi}=-2 L \phi-\gamma\left((1+\eta) \phi^{2}+1\right) \text { with } \phi(0)=\frac{1}{\rho^{*}}
$$

where $\eta>0, \rho^{*} \in(\rho, 1)$. The time $\widetilde{\mathcal{T}}\left(\rho^{*}, \gamma, L, \eta\right)$ it takes for $\phi$ to decrease from $1 / \rho^{*}$ to $\rho^{*}$ is a continuous function in $\eta$ and $\rho^{*}$ which decreases with both increasing $\eta$ and $\rho^{*}$ as long as $\rho^{*} \leq 1$ (by invoking the comparison principle). Moreover, we have that $\widetilde{\mathcal{T}}(\rho, \gamma, L, 0)=\mathcal{T}(\rho, \gamma, L)$, as a consequence $\widetilde{\mathcal{T}}\left(\rho^{*}, \gamma, L, \eta\right) \leq$ $\mathcal{T}(\rho, \gamma, L)$. Based on these facts, for any $\tau^{*}<\mathcal{T}(\rho, \gamma, L)$ we can always find $\rho^{*}$ sufficiently close to $\rho$ with $\rho^{*}>\rho$ and $\eta$ sufficiently small such that $\tau^{*}<\widetilde{\mathcal{T}}\left(\rho^{*}, \gamma, L, \eta\right)$. In the following, we take $\eta \in\left(0,\left(\rho^{*} / \rho\right)^{2}-1\right)$.

The following claim follows from Claim 1 in [2] and the developments above.

Claim 1: For all $\tau_{1} \in\left[0, \tau^{*}\right], \phi\left(\tau_{1}\right) \in\left[\rho^{*},\left(1 / \rho^{*}\right)\right]$.

For the sake of convenience, we introduce $q:=\left(q_{x}, q_{e}, \kappa, \tau_{1}\right.$, $\left.\tau_{2}\right) \in \mathcal{R}$ where $\mathcal{R}:=\mathcal{R}_{x} \times \mathcal{R}_{e} \times \mathbb{Z}_{\geq 0} \times \mathbb{R}_{\geq 0}^{2}$ and write system (11) as

$$
\dot{q}=F(q, w) \text { for } q \in C, \quad q^{+}=G(q) \text { for } q \in D
$$

where $C:=\left\{q \in \mathcal{R}: \tau \in\left[0, \tau^{*}\right]\right\}$ and $D:=\{q \in \mathcal{R}: \tau \in$ $\left.\left[v, \tau^{*}\right]\right\}$. We define, for all $q \in C \cup D \cup G(D)$

$$
U(q):=V\left(q_{x}\right)+\gamma \phi\left(\tau_{1}\right) W^{2}\left(\kappa, q_{e}\right) .
$$


According to Remark 2.3 in [23] and Assumptions 1 and 3, we have that

$$
\underline{\alpha}_{U}(|(\xi, e)|) \leq U(q) \leq \bar{\alpha}_{U}\left(\left|\left(q_{x}, q_{e}\right)\right|\right)
$$

with $\underline{\alpha}_{U}: s \mapsto \min \left\{\underline{\alpha}_{V}(s / 2), \rho^{*} \underline{\alpha}_{W}(s / 2)\right\} \in \mathcal{K}_{\infty}$ and $\bar{\alpha}_{U}$ : $s \mapsto \bar{\alpha}_{V}(s)+\left(1 / \rho^{*}\right) \bar{\alpha}_{W}(s) \in \mathcal{K}_{\infty}$.

In view of (41) and since $q_{x}^{+}=q_{x}$

$$
\begin{aligned}
U(G(q)) & =V\left(q_{x}\right)+\gamma \phi(0) W^{2}\left(\kappa+1, h\left(\kappa, q_{e}\right)\right) \\
& =V\left(q_{x}\right)+\gamma \frac{1}{\rho^{*}} W^{2}\left(\kappa+1, h\left(\kappa, q_{e}\right)\right) .
\end{aligned}
$$

Using Assumption 1 (we omit the arguments of $V$ and $W$ in the following for the sake of simplicity), we obtain

$$
\begin{aligned}
U(G(q)) \leq V+\gamma \frac{1}{\rho^{*}}\left(\rho W+\mu^{d}\left(\left|e_{d}\right|\right)+\mu^{f f}\left(\left|e_{f f}\right|\right)\right)^{2} \\
=V+\gamma \frac{1}{\rho^{*}}\left(\rho^{2} W^{2}+2 \rho W\left(\mu^{d}\left(\left|e_{d}\right|\right)+\mu^{f f}\left(\left|e_{f f}\right|\right)\right)\right. \\
\left.\quad+\left(\mu^{d}\left(\left|e_{d}\right|\right)+\mu^{f f}\left(\left|e_{f f}\right|\right)\right)^{2}\right)
\end{aligned}
$$

We are going to upper bound the right-hand side of the above equation using the following inequalities (we utilize that $2 a b \leq$ $a^{2}+b^{2}$ for $a, b \in \mathbb{R}$ )

$$
\begin{aligned}
\left(\mu^{d}\left(\left|e_{d}\right|\right)+\mu^{f f}\left(\left|e_{f f}\right|\right)\right)^{2}= & \mu^{d}\left(\left|e_{d}\right|\right)^{2}+\mu^{f f}\left(\left|e_{f f}\right|\right)^{2} \\
& +2 \mu^{d}\left(\left|e_{d}\right|\right) \mu^{f f}\left(\left|e_{f f}\right|\right) \\
\leq & 2 \mu^{d}\left(\left|e_{d}\right|\right)^{2}+2 \mu^{f f}\left(\left|e_{f f}\right|\right)^{2}
\end{aligned}
$$

and (using that $2 a b \leq(\eta / 2) a^{2}+(2 / \eta) b^{2}$ for $a, b \in \mathbb{R}$ )

$$
\begin{aligned}
2 \rho W & \left(\mu^{d}\left(\left|e_{d}\right|\right)+\mu^{f f}\left(\left|e_{f f}\right|\right)\right) \\
& =2 \rho W \mu^{d}\left(\left|e_{d}\right|\right)+2 \rho W \mu^{f f}\left(\left|e_{f f}\right|\right) \\
& \leq \frac{\eta}{2} \rho^{2} W^{2}+\frac{2}{\eta} \mu^{d}\left(\left|e_{d}\right|\right)^{2}+\frac{\eta}{2} \rho^{2} W^{2}+\frac{2}{\eta} \mu^{f f}\left(\left|e_{f f}\right|\right)^{2} \\
& =\eta \rho^{2} W^{2}+\frac{2}{\eta} \mu^{d}\left(\left|e_{d}\right|\right)^{2}+\frac{2}{\eta} \mu^{f f}\left(\left|e_{f f}\right|\right)^{2}
\end{aligned}
$$

As a consequence, we obtain the following bound on $U(G(q))$ from (46)

$$
\begin{aligned}
& U(G(q)) \\
& \begin{aligned}
\leq V+\gamma \frac{1}{\rho^{*}}( & \rho^{2} W^{2}+\eta \rho^{2} W^{2}+\frac{2}{\eta} \mu^{d}\left(\left|e_{d}\right|\right)^{2} \\
& +\frac{2}{\eta} \mu^{f f}\left(\left|e_{f f}\right|\right)^{2} \\
& \left.+2 \mu^{d}\left(\left|e_{d}\right|\right)^{2}+2 \mu^{f f}\left(\left|e_{f f}\right|\right)^{2}\right) \\
=V+\gamma \frac{1}{\rho^{*}}( & (1+\eta) \rho^{2} W^{2} \\
+ & \left.+2\left(1+\frac{1}{\eta}\right)\left(\mu^{d}\left(\left|e_{d}\right|\right)^{2}+\mu^{f f}\left(\left|e_{f f}\right|\right)^{2}\right)\right) .
\end{aligned}
\end{aligned}
$$

Denote $\sigma_{U}^{d}(s):=\gamma\left(2 / \rho^{*}\right)(1+(1 / \eta)) \mu^{d}(s)^{2}$ and $\sigma_{U}^{f f}(s):=\gamma(2 /$ $\left.\rho^{*}\right)(1+(1 / \eta)) \mu^{f f}(s)^{2}$ for $s \geq 0$ and notice that $\left(1 / \rho^{*}\right)(1+$

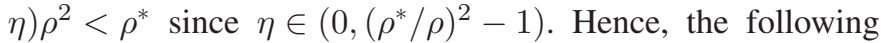
holds according to Claim 1

$$
\begin{aligned}
U(G(q)) & \leq V+\gamma \rho^{*} W^{2}+\sigma_{U}^{d}\left(\left|e_{d}\right|\right)+\sigma_{U}^{f f}\left(\left|e_{f f}\right|\right) \\
& \leq V+\gamma \phi\left(\tau_{1}\right) W^{2}+\sigma_{U}^{d}\left(\left|e_{d}\right|\right)+\sigma_{U}^{f f}\left(\left|e_{f f}\right|\right) \\
& =U(q)+\sigma_{U}^{d}\left(\left|e_{d}\right|\right)+\sigma_{U}^{f f}\left(\left|e_{f f}\right|\right) .
\end{aligned}
$$

We now study the dynamics of $U$ on flows. ${ }^{7}$ For all $\kappa \in$ $\mathbb{Z}_{\geq 0}, \tau_{1} \in\left[0, \tau^{*}\right], \tau_{2} \in \mathbb{R}_{\geq 0}, w \in \mathbb{R}^{n_{w}}$ and almost all $\left(q_{x}, q_{e}\right) \in$ $\mathcal{R}_{x} \times \mathcal{R}_{e}$, we have that, in view of Assumptions 2-3 and (41)

$$
\begin{aligned}
\langle\nabla U & (q), F(q, w)\rangle \\
\leq & -\varepsilon V-\varepsilon W^{2}-H^{2}\left(q_{x}\right)+\gamma^{2} W^{2}+\sigma^{d}\left(\left|e_{d}\right|\right) \\
& +\sigma^{f f}\left(\left|e_{f f}\right|\right)+\sigma^{w}(|w|) \\
& +\gamma\left(-2 L \phi-\gamma\left((1+\eta) \phi^{2}+1\right)\right) W^{2} \\
& +2 \gamma \phi W\left(L W+H\left(q_{x}\right)+\nu^{d}\left(\left|e_{d}\right|\right)+\nu^{f f}\left(\left|e_{f f}\right|\right)\right. \\
\quad & \left.\quad+\nu^{w}(|w|)\right) \\
= & -\varepsilon V-\varepsilon W^{2}-H^{2}\left(q_{x}\right)+\sigma^{d}\left(\left|e_{d}\right|\right) \\
& +\sigma^{f f}\left(\left|e_{f f}\right|\right)+\sigma^{w}(|w|) \\
& +\gamma\left(-2 L \phi-\gamma(1+\eta) \phi^{2}\right) W^{2} \\
& +2 \gamma \phi W\left(L W+H\left(q_{x}\right)+\nu^{d}\left(\left|e_{d}\right|\right)+\nu^{f f}\left(\left|e_{f f}\right|\right)\right. \\
& \left.\quad+\nu^{w}(|w|)\right) .
\end{aligned}
$$

We are going to upper bound the term on the last line of the inequality above. Using that $2 a b \leq a^{2}+b^{2}$ for $a, b \in \mathbb{R}$, we obtain $2 \gamma \phi W H\left(q_{x}\right) \leq \gamma^{2} \phi^{2} W^{2}+H^{2}\left(q_{x}\right)$ and, using that $2 a b \leq(\eta / 3) a^{2}+(3 / \eta) b^{2}$ for $a, b \in \mathbb{R}$, yields

$$
\begin{aligned}
2 \gamma \phi W & \left(\nu^{d}\left(\left|e_{d}\right|\right)+\nu^{f f}\left(\left|e_{f f}\right|\right)+\nu^{w}(|w|)\right) \\
= & 2 \gamma \phi W \nu^{d}\left(\left|e_{d}\right|\right)+2 \gamma \phi W \nu^{f f}\left(\left|e_{f f}\right|\right)+2 \gamma \phi W \nu^{w}(|w|) \\
\leq & \frac{\eta}{3} \gamma^{2} \phi^{2} W^{2}+\frac{3}{\eta} \nu^{d}\left(\left|e_{d}\right|\right)^{2}+\frac{\eta}{3} \gamma^{2} \phi^{2} W^{2}+\frac{3}{\eta} \nu^{f f}\left(\left|e_{f f}\right|\right)^{2} \\
& \quad+\frac{\eta}{3} \gamma^{2} \phi^{2} W^{2}+\frac{3}{\eta} \nu^{w}(|w|)^{2} \\
= & \eta \gamma^{2} \phi^{2} W^{2}+\frac{3}{\eta}\left(\nu^{d}\left(\left|e_{d}\right|\right)^{2}+\nu^{f f}\left(\left|e_{f f}\right|\right)^{2}+\nu^{w}(|w|)^{2}\right) .
\end{aligned}
$$

Going back to (51), we derive that

$$
\begin{aligned}
\langle\nabla U & (q), F(q, w)\rangle \\
\leq & -\varepsilon V-\varepsilon W^{2}-H^{2}\left(q_{x}\right)+\sigma^{d}\left(\left|e_{d}\right|\right) \\
& +\sigma^{f f}\left(\left|e_{f f}\right|\right)+\sigma^{w}(|w|) \\
& +\gamma\left(-2 L \phi-\gamma(1+\eta) \phi^{2}\right) W^{2} \\
& +2 \gamma \phi L W^{2}+\gamma^{2} \phi^{2} W^{2}+H^{2}\left(q_{x}\right) \\
& +\eta \gamma^{2} \phi^{2} W^{2}+\frac{3}{\eta}\left(\nu^{d}\left(\left|e_{d}\right|\right)^{2}+\nu^{f f}\left(\left|e_{f f}\right|\right)^{2}\right. \\
& \left.+\nu^{w}(|w|)^{2}\right) \\
= & -\varepsilon V-\varepsilon W^{2}+\sigma^{d}\left(\left|e_{d}\right|\right)+\sigma^{f f}\left(\left|e_{f f}\right|\right) \\
& +\sigma^{w}(|w|)+\frac{3}{\eta}\left(\nu^{d}\left(\left|e_{d}\right|\right)^{2}+\nu^{f f}\left(\left|e_{f f}\right|\right)^{2}\right. \\
& \left.+\nu^{w}(|w|)^{2}\right) .
\end{aligned}
$$

\footnotetext{
${ }^{7}$ We consider $\langle\nabla U(q), F(q, w)\rangle$ with some abuse of notation since $U$ is not (almost everywhere) differentiable a priori with respect to $\kappa$. However, this is justified by the fact that $\dot{\kappa}=0$, see (11).
} 
Therefore, there exists $\tilde{\varepsilon}>0$ according to Claim 1 (take $\tilde{\varepsilon} \in$ $\left.\left(0, \varepsilon \min \left\{1,\left(\rho^{*} / \gamma\right)\right\}\right)\right)$ such that

$$
\begin{array}{r}
\langle\nabla U(q), F(q, w)\rangle \leq-\tilde{\varepsilon} U(q)+\varsigma_{U}^{d}\left(\left|e_{d}\right|\right)+\varsigma_{U}^{f f}\left(\left|e_{f f}\right|\right) \\
+\varsigma_{U}^{w}(|w|)
\end{array}
$$

with $\quad \varsigma_{U}^{d}(s):=\sigma^{d}(s)+(3 / \eta) \nu^{d}(s)^{2}, \quad \varsigma_{U}^{f f}(s):=\sigma^{f f}(s)+$ $(3 / \eta) \nu^{f f}(s)^{2}, \varsigma_{U}^{w}(s):=\sigma^{w}(s)+(3 / \eta) \nu^{w}(s)^{2}$ for $s \geq 0$.

Let $(q, w)$ be a solution pair to system (42). From (54), by invoking standard comparison principles for continuous-time systems, we obtain that, for $\left(t_{1}, 0\right) \in \operatorname{dom} q$

$$
\begin{aligned}
U\left(q\left(t_{1}, 0\right)\right) \leq & \exp \left(-\tilde{\varepsilon} t_{1}\right) U(q(0,0))+\tilde{\varepsilon}^{-1}\left(\varsigma_{U}^{d}\left(\left\|e_{d}\right\|_{\left(t_{1}, 0\right)}\right)\right. \\
& \left.+\varsigma_{U}^{f f}\left(\left\|e_{f f}\right\|_{\left(t_{1}, 0\right)}\right)+\varsigma_{U}^{w}\left(\|w\|_{\left(t_{1}, 0\right)}\right)\right) .
\end{aligned}
$$

On the other hand, from (50), for $\left(t_{1}, 1\right) \in \operatorname{dom} q$

$$
\begin{aligned}
U\left(q\left(t_{1}, 1\right)\right) \leq U\left(q\left(t_{1}, 0\right)\right)+\sigma_{U}^{d} & \left(\left\|e_{d}\right\|_{\left(t_{1}, 0\right)}\right) \\
& +\sigma_{U}^{f f}\left(\left\|e_{f f}\right\|_{\left(t_{1}, 0\right)}\right) .
\end{aligned}
$$

By induction, we have that, for $(t, j) \in \operatorname{dom} q$

$$
\begin{aligned}
& U(q(t, j)) \\
& \leq \exp (-\tilde{\varepsilon} t) U(q(0,0))+\bar{\sigma}_{U}^{w}\left(\|w\|_{(t, j)}\right) \\
& \quad+\left(\bar{\sigma}_{U}^{d}\left(\left\|e_{d}\right\|_{(t, j)}\right)+\bar{\sigma}_{U}^{f f}\left(\left\|e_{f f}\right\|_{(t, j)}\right)\right) \\
& \quad \times \sum_{k=0}^{j-1} \exp (-\tilde{\varepsilon} v)^{k} \\
& \leq \exp (-\tilde{\varepsilon} t) U(q(0,0))+\bar{\sigma}_{U}^{w}\left(\|w\|_{(t, j)}\right) \\
& \quad+\left(\bar{\sigma}_{U}^{d}\left(\left\|e_{d}\right\|_{(t, j)}\right)+\bar{\sigma}_{U}^{f f}\left(\left\|e_{f f}\right\|_{(t, j)}\right)\right) \frac{1}{1-\exp (-\tilde{\varepsilon} v)}
\end{aligned}
$$

where $\bar{\sigma}_{U}^{d}(s)=\sigma_{U}^{d}(s)+\tilde{\varepsilon}^{-1} \varsigma_{U}^{d}(s), \bar{\sigma}_{U}^{f f}(s)=\sigma_{U}^{f f}(s)+\tilde{\varepsilon}^{-1} \varsigma_{U}^{f f}(s)$ and $\bar{\sigma}_{U}^{w}(s)=\tilde{\varepsilon}^{-1} \varsigma_{U}^{w}(s)$ for $s \geq 0$. On the other hand, using (44) in (57), we obtain $|(\xi(t, j), e(t, j))| \leq \underline{\alpha}_{U}^{-1}\left(\exp (-\tilde{\varepsilon} t) \bar{\alpha}_{U}\left(\mid\left(q_{x}(0\right.\right.\right.$, $\left.\left.0), q_{e}(0,0)\right) \mid\right)+\bar{\sigma}_{U}^{w}\left(\|w\|_{(t, j)}\right)+\left(\bar{\sigma}_{U}^{d}\left(\left\|e_{d}\right\|_{(t, j)}\right)+\bar{\sigma}_{U}^{f f}\left(\left\|e_{f f}\right\|_{(t, j)}\right)\right)$ $(1 /(1-\exp (-\tilde{\varepsilon} v))))$. By using several times the fact that $\chi(a+b) \leq \chi(2 a)+\chi(2 b)$ for any $\chi \in \mathcal{K}_{\infty}$ and $a, b \geq 0$, we obtain the desired result (17).

We now prove the last part of Theorem 1 . We only consider $\delta^{d}$ without loss of generality and let $s \geq 0$. We have that (17) holds with $\delta^{d}(s)=\underline{\alpha}_{U}^{-1}\left((4 /(1-\exp (-\tilde{\varepsilon} v))) \bar{\sigma}_{U}^{d}(s)\right)$. It has to be noted that any upper bound of $\underline{\alpha}_{U}^{-1}((4 /(1-$ $\left.\exp (-\tilde{\varepsilon} v))) \bar{\sigma}_{U}^{d}(s)\right)$ can be taken to be $\delta^{d}$ in (17). Thus, we will derive upper bounds for $\delta^{d}$ which are of the desired form. Using the definition of $\bar{\sigma}_{U}^{d}$ given after (57), we obtain

$$
\delta^{d}(s)=\underline{\alpha}_{U}^{-1}\left(\frac{4}{1-\exp (-\tilde{\varepsilon} v)}\left(\sigma_{U}^{d}(s)+\tilde{\varepsilon}^{-1} \varsigma_{U}^{d}(s)\right)\right)
$$

which gives, in view of the definition of $\sigma_{U}^{d}$ and $\varsigma_{U}^{d}$, respectively, given after (49) and (54)

$$
\begin{array}{r}
\delta^{d}(s)=\underline{\alpha}_{U}^{-1}\left(\frac { 4 } { 1 - \operatorname { e x p } ( - \tilde { \varepsilon } v ) } \left[\gamma \frac{2}{\rho^{*}}\left(1+\frac{1}{\eta}\right) \mu^{d}(s)^{2}\right.\right. \\
\left.\left.+\tilde{\varepsilon}^{-1}\left(\sigma^{d}(s)+\frac{3}{\eta} \nu^{d}(s)^{2}\right)\right]\right) .
\end{array}
$$

The function $\delta^{d}$ depends on the MATI $\tau^{*}$ although that is not obvious from (59) because this dependence is hidden in the constants $\rho^{*}$ and $\eta$. Thus, we will remove the dependence of $\delta^{d}$ on $\rho^{*}$. We know that $\rho^{*}>\rho$. Therefore, noting that $\underline{\alpha}_{U}(s)=$ $\min \left\{\underline{\alpha}_{V}(s / 2), \rho^{*} \underline{\alpha}_{W}(s / 2)\right\} \geq \min \left\{\underline{\alpha}_{V}(s / 2), \rho \underline{\alpha}_{W}(s / 2)\right\}=:$ $\underline{\tilde{\alpha}}_{U}(s)$ (in view of the definition of $\underline{\alpha}_{U}$ given below (44)) and since we are working with strictly increasing functions

$$
\begin{array}{r}
\delta^{d}(s) \leq \underline{\tilde{\alpha}}_{U}^{-1}\left(\frac { 4 } { 1 - \operatorname { e x p } ( - \tilde { \varepsilon } v ) } \left[\gamma \frac{2}{\rho}\left(1+\frac{1}{\eta}\right) \mu^{d}(s)^{2}\right.\right. \\
\left.\left.+\tilde{\varepsilon}^{-1}\left(\sigma^{d}(s)+\frac{3}{\eta} \nu^{d}(s)^{2}\right)\right]\right) .
\end{array}
$$

The constant $\tilde{\varepsilon}$ satisfies $\tilde{\varepsilon} \in\left(0, \varepsilon \min \left\{1,\left(\rho^{*} / \gamma\right)\right\}\right)$, see above (54). However, since $\rho^{*}>\rho$, we can take $\tilde{\varepsilon} \in(0, \varepsilon \min \{1,(\rho)$ $\gamma)\})$. In that way, (60) becomes independent of $\rho^{*}$. We write $\eta=\theta\left(\tau^{*}\right)^{-1}$ for some strictly positive function $\theta: \mathbb{R}_{\geq 0} \rightarrow \mathbb{R}_{>0}$, in that way (60) becomes

$$
\begin{aligned}
\delta^{d}(s) \leq \underline{\tilde{\alpha}}_{U}^{-1} & \left(\frac { 4 } { 1 - \operatorname { e x p } ( - \varepsilon \operatorname { m i n } \{ 1 , \frac { \rho } { \gamma } \} v ) } \left[\gamma \frac{2}{\rho}\left(1+\theta\left(\tau^{*}\right)\right) \mu^{d}(s)^{2}\right.\right. \\
& \left.\left.+\frac{1}{\varepsilon \min \left\{1, \frac{\rho}{\gamma}\right\}}\left(\sigma^{d}(s)+3 \theta\left(\tau^{*}\right) \nu^{d}(s)^{2}\right)\right]\right) \cdot(61)
\end{aligned}
$$

As a consequence, by applying several times the property $\chi(a+b) \leq \chi(2 a)+\chi(2 b)$ for any $\chi \in \mathcal{K}_{\infty}$ and $a, b \geq 0$, we obtain that $\delta^{d}(s) \leq \psi\left(v^{-1}\right)\left(\tilde{\delta}(s)+\varphi\left(\tau^{*}\right) \bar{\delta}(s)\right) \leq\left(1+\varphi\left(\tau^{*}\right)\right)$ $\psi\left(v^{-1}\right) \delta(s)$, where $\tilde{\delta}, \bar{\delta} \in \mathcal{K}_{\infty}, \varphi: \mathbb{R}_{\geq 0} \rightarrow \mathbb{R}_{>0}$ and $\delta(s):=$ $\max \{\tilde{\delta}(s), \bar{\delta}(s)\}$.

Sketch of Proof of Proposition 1: Property (17) holds according to Theorem 1. We then just have to use (18) in (17) and (19) and to combine the obtained inequalities to deduce that (20) holds on the domain of the solution.

Proof of Proposition 2: We define the function $W: \mathbb{Z}_{\geq 0} \times$ $\mathbb{R}^{n_{e}} \rightarrow \mathbb{R}_{\geq 0}$ as $W:(\kappa, e) \mapsto \mathbf{W}\left(\kappa, e_{p}-e_{d}, 0, e_{f b}, 0\right)$, which is locally Lipschitz in view of item (ii) of Proposition 2. From (22), we deduce that the first line of (13) is ensured with $\underline{\alpha}_{W}(s)=\underline{\boldsymbol{\alpha}}_{\mathbf{W}}(s)$ and $\bar{\alpha}_{W}(s)=\overline{\boldsymbol{\alpha}}_{\mathbf{W}}(s)$ for $s \geq 0$. Moreover, for system (11) we have that $W\left(\kappa^{+}, e^{+}\right)=\mathbf{W}\left(\kappa^{+}, e_{p}^{+}-\right.$ $\left.e_{d}^{+}, 0, e_{f b}^{+}, 0\right)-\mathbf{W}\left(\kappa^{+}, \mathbf{e}^{+}\right)+\mathbf{W}\left(\kappa^{+}, \mathbf{e}^{+}\right)$. Using $\kappa^{+}=\kappa+1$ from (11) and (23), we obtain

$$
\begin{aligned}
W\left(\kappa^{+}, e^{+}\right) \leq & \mathbf{W}\left(\kappa^{+}, e_{p}^{+}-e_{d}^{+}, 0, e_{f b}^{+}, 0\right)-\mathbf{W}\left(\kappa^{+}, \mathbf{e}^{+}\right) \\
& +\boldsymbol{\rho} \mathbf{W}(\kappa, \mathbf{e}) \\
= & \mathbf{W}\left(\kappa^{+}, e_{p}^{+}-e_{d}^{+}, 0, e_{f b}^{+}, 0\right)-\mathbf{W}\left(\kappa^{+}, \mathbf{e}^{+}\right) \\
& +\boldsymbol{\rho} \mathbf{W}(\kappa, \mathbf{e})-\boldsymbol{\rho} \mathbf{W}\left(\kappa, e_{p}-e_{d}, 0, e_{f b}, 0\right) \\
& +\boldsymbol{\rho} \mathbf{W}\left(\kappa, e_{p}-e_{d}, 0, e_{f b}, 0\right) .
\end{aligned}
$$


Since item (ii) of Proposition 2 is satisfied and by recalling that $\mathbf{e}=\left(e_{p}, e_{d}, e_{f b}, e_{f f}\right)$, we have that $\mathbf{W}\left(\kappa, e_{p}-e_{d}, 0, e_{f b}, 0\right)-$ $\mathbf{W}(\kappa, \mathbf{e})=\mathbf{W}\left(\kappa, e_{p}-e_{d}, 0, e_{f b}, 0\right)-\mathbf{W}\left(\kappa, e_{p}, e_{d}, e_{f b}, e_{f f}\right) \leq$ $M\left|\left(e_{d}, e_{d}, e_{f f}\right)\right|$ using the mean value theorem (since $\mathbf{W}$ is locally Lipschitz in e). Similarly, we derive $\mathbf{W}\left(\kappa^{+}, e_{p}^{+}-e_{d}^{+}, 0\right.$, $\left.e_{f b}^{+}, 0\right)-\mathbf{W}\left(\kappa^{+}, \mathbf{e}^{+}\right) \leq M\left|\left(e_{d}^{+}, e_{d}^{+}, e_{f f}^{+}\right)\right|$. In view of item (i) of Proposition 2, we know that $\left|e_{d}^{+}\right| \leq\left|e_{d}\right|$ and $\left|e_{f f}^{+}\right| \leq\left|e_{f f}\right|$; consequently $\mathbf{W}\left(\kappa^{+}, e_{p}^{+}-e_{d}^{+}, 0, e_{f b}^{+}, 0\right)-\mathbf{W}\left(\kappa^{+}, \mathbf{e}^{+}\right) \leq M \mid\left(e_{d}\right.$, $\left.e_{d}, e_{f f}\right) \mid$. As a consequence, in view of (62), we obtain

$$
\begin{aligned}
W\left(\kappa^{+}, e^{+}\right) \leq & M\left|\left(e_{d}, e_{d}, e_{f f}\right)\right|+\boldsymbol{\rho} M\left|\left(e_{d}, e_{d}, e_{f f}\right)\right| \\
& +\boldsymbol{\rho} \mathbf{W}\left(\kappa, e_{p}-e_{d}, 0, e_{f b}, 0\right) \\
\leq & \boldsymbol{\rho} W(\kappa, e)+2 M(1+\boldsymbol{\rho})\left|e_{d}\right|+M(1+\boldsymbol{\rho})\left|e_{f f}\right|
\end{aligned}
$$

and the second line of (13) is verified with $\rho=\rho, \mu^{d}(s)=$ $2 M(1+\boldsymbol{\rho}) s$ and $\mu^{f f}(s)=M(1+\boldsymbol{\rho}) s$ for $s \geq 0$.

Proof of Proposition 3: For the RR protocol, we can write (see (9) or Section III in [4])

$$
\begin{aligned}
h_{p}\left(\kappa, e_{p}\right) & =\left(\mathbb{I}-\Psi_{p}(\kappa)\right) e_{p} \\
h_{d}\left(\kappa, e_{d}\right) & =\left(\mathbb{I}-\Psi_{d}(\kappa)\right) e_{d} \\
h_{f b}\left(\kappa, e_{f b}\right) & =\left(\mathbb{I}-\Psi_{f b}(\kappa)\right) e_{f b}
\end{aligned}
$$

where $\Psi_{p}, \Psi_{d}, \Psi_{f b}$ are diagonal matrices whose diagonals are composed of 0 and 1.

We consider $W(\kappa, e)=\sqrt{\sum_{i=\kappa}^{\infty}|\phi(i, \kappa, e)|^{2}} \quad$ where $\phi(i, \kappa, e)$ is the solution to the following system at time $i$ starting at time $\kappa$ with initial condition $e$ :

$$
\begin{aligned}
\bar{e}^{+} & =\left(\begin{array}{c}
h_{p}\left(\kappa, e_{\xi}\right) \\
h_{f b}\left(\kappa, e_{f b}\right)
\end{array}\right)=\left(\begin{array}{c}
\left(\mathbb{I}-\Psi_{p}(\kappa)\right) e_{\xi} \\
\left(\mathbb{I}-\Psi_{f b}(\kappa)\right) e_{f b}
\end{array}\right) \\
& =: \bar{h}_{e}(\kappa, e) .
\end{aligned}
$$

By following the same lines as in the proof of Proposition 4 in [4] since system (65) is dead-beat stable in $\ell$ steps and $|\phi(i, \kappa, e)| \leq|e|$ for all $i \geq \kappa \geq 0$ and $e \in \mathbb{R}^{n_{e}}$, we deduce that the first line of (13) holds with $\underline{\alpha}_{W}(s)=s, \bar{\alpha}_{W}(s)=\sqrt{\ell} s$ for $s \geq 0$ in view of Proposition 4 in [4]. We now show that the second line of (13) is guaranteed: $W\left(\kappa+1, h_{e}\right.$ $\left.\left(\kappa, e, e_{d}, e_{f f}\right)\right)=\sqrt{\sum_{i=\kappa+1}^{\infty}\left|\phi\left(i, \kappa+1, h_{e}\left(\kappa, e, e_{d}, e_{f f}\right)\right)\right|^{2}}=$ $\sqrt{\sum_{i=\kappa+1}^{\infty}\left|\phi\left(i, \kappa+1, \bar{h}_{e}(\kappa, e)+\Delta h_{e}\left(\kappa, e, e_{d}, e_{f f}\right)\right)\right|^{2}}$ where $h_{e}$ is introduced in Section IV and $\Delta h_{e}\left(\kappa, e, e_{d}, e_{f f}\right)=h_{e}(\kappa, e$, $\left.e_{d}, e_{f f}\right)-\bar{h}_{e}(\kappa, e)$. Due to the linearity of $\phi$ in its third argument in view of (65), we have that $\phi\left(i, \kappa+1, \bar{h}_{e}(\kappa, e)+\right.$ $\left.\Delta h_{e}\left(\kappa, e, e_{d}, e_{f f}\right)\right)=\phi\left(i, \kappa+1, \bar{h}_{e}(\kappa, e)\right)+\phi\left(i, \kappa+1, \Delta h_{e}(\kappa\right.$, $\left.\left.e, e_{d}, e_{f f}\right)\right)$. In that way, we derive, using that $\sqrt{a+b} \leq$ $\sqrt{a}+\sqrt{b}$ for $a, b \in \mathbb{R}_{\geq 0}$

$$
\begin{aligned}
W & \left(\kappa+1, h_{e}\left(\kappa, e, e_{d}, e_{f f}\right)\right) \\
= & \sqrt{\sum_{i=\kappa+1}^{\infty}\left|\phi\left(i, \kappa+1, \bar{h}_{e}(\kappa, e)\right)\right|^{2}} \\
& +\sqrt{\sum_{i=\kappa+1}^{\infty}\left|\phi\left(i, \kappa+1, \Delta h_{e}\left(\kappa, e, e_{d}, e_{f f}\right)\right)\right|^{2}} .
\end{aligned}
$$

Denote $R(\kappa, e)=\sum_{i=\kappa}^{\infty}|\phi(i, \kappa, e)|^{2}$; using the fact that $\phi(i, i, e)=e$

$$
\begin{aligned}
R\left(\kappa+1, \bar{h}_{e}(\kappa, e)\right) & =\sum_{i=\kappa+1}^{\infty}\left|\phi\left(i, \kappa+1, \bar{h}_{e}(\kappa, e)\right)\right|^{2} \\
& =\sum_{i=\kappa}^{\infty}|\phi(i, \kappa, e)|^{2}-|e|^{2}=R(\kappa, e)-|e|^{2}
\end{aligned}
$$

Now, we observe that $R(\kappa, e)=W^{2}(\kappa, e) \leq \ell|e|^{2}$ and thus $R\left(\kappa+1, \bar{h}_{e}(\kappa, e)\right) \leq R(\kappa, e)-(1 / \ell) R(\kappa, e)=((\ell-1) / \ell) R(\kappa, e)$ which implies

$$
\begin{aligned}
W\left(\kappa+1, \bar{h}_{e}(\kappa, e)\right) & =\sqrt{\sum_{i=\kappa+1}^{\infty}\left|\phi\left(i, \kappa+1, \bar{h}_{e}(\kappa, e)\right)\right|^{2}} \\
& \leq \sqrt{\frac{\ell-1}{\ell} W(\kappa, e) .}
\end{aligned}
$$

On the other hand, we notice that $\mid \phi\left(i, \kappa+1, \Delta h_{e}\left(\kappa, e, e_{d}\right.\right.$, $\left.\left.e_{f f}\right)\right)|\leq| \Delta h_{e}\left(\kappa, e, e_{d}, e_{f f}\right) \mid$ in view of (64) and the fact that $\Psi_{p}$ and $\Psi_{d}$ are diagonal matrices whose diagonals are composed of 0 and 1 . As a consequence, we have that

$$
\begin{aligned}
h_{e}\left(\kappa, e, e_{d}, e_{f f}\right) & =\left(\begin{array}{c}
\left(\mathbb{I}-\Psi_{p}(\kappa)\right) e_{p}-\left(\mathbb{I}-\Psi_{d}(\kappa)\right) e_{d} \\
\left(\mathbb{I}-\Psi_{f b}(\kappa)\right) e_{f b}
\end{array}\right) \\
& =\left(\begin{array}{c}
\left(\mathbb{I}-\Psi_{p}(\kappa)\right) e_{\xi}+\left(\Psi_{d}(\kappa)-\Psi_{p}(\kappa)\right) e_{d} \\
\left(\mathbb{I}-\Psi_{f b}(\kappa)\right) e_{f b}
\end{array}\right) .
\end{aligned}
$$

Hence

$$
\begin{aligned}
\Delta h_{e}\left(\kappa, e, e_{d}, e_{f f}\right)= & \left(\begin{array}{c}
\left(\mathbb{I}-\Psi_{p}(\kappa)\right) e_{\xi}+\left(\Psi_{d}(\kappa)-\Psi_{p}(\kappa)\right) e_{d} \\
\left(\mathbb{I}-\Psi_{f b}(\kappa)\right) e_{f b}
\end{array}\right) \\
& -\left(\begin{array}{c}
\left(\mathbb{I}-\Psi_{p}(\kappa)\right) e_{\xi} \\
\left(\mathbb{I}-\Psi_{f b}(\kappa)\right) e_{f b}
\end{array}\right) \\
= & \left(\begin{array}{c}
\left(\Psi_{d}(\kappa)-\Psi_{p}(\kappa)\right) e_{d} \\
0
\end{array}\right) .
\end{aligned}
$$

Therefore $\left|\phi\left(i, \kappa+1, \Delta h_{e}\left(\kappa, e, e_{d}, e_{f f}\right)\right)\right| \leq \mid\left(\Psi_{d}(\kappa)-\Psi_{p}(\kappa)\right)$ $e_{d}|\leq| \Psi_{d}(\kappa)-\Psi_{p}(\kappa)|| e_{d} \mid$. Since $\Psi_{p}(\kappa)$ and $\Psi_{d}(\kappa)$ are diagonal matrices whose diagonal components are 0 or 1 , we deduce that $\left|\Psi_{p}(\kappa)-\Psi_{d}(\kappa)\right| \leq 1$. In that way, we obtain that $\left|\Delta h_{e}\left(\kappa, e, e_{d}, e_{f f}\right)\right| \leq\left|e_{d}\right|$. As a consequence, $\mid \phi(i, \kappa+1$, $\left.\Delta h_{e}\left(\kappa, e, e_{d}, e_{f f}\right)\right)|\leq| e_{d} \mid$. Combining this point with the fact that system (65) is dead-beat stable in $\ell$ steps, we obtain

$$
\sqrt{\sum_{i=\kappa+1}^{\infty}\left|\phi\left(i, \kappa+1, \Delta h_{e}\left(\kappa, e, e_{d}, e_{f f}\right)\right)\right|^{2}} \leq \sqrt{\ell}\left|e_{d}\right| .
$$

Therefore, in view of (66), (68) and (70), $W\left(\kappa+1, h_{e}\left(\kappa, e, e_{d}\right.\right.$, $\left.\left.e_{f f}\right)\right) \leq \sqrt{((\ell-1) / \ell)} W(i, e)+\sqrt{\ell}\left|e_{d}\right|$. Hence the second line of (13) holds with $\rho=\sqrt{(\ell-1) / \ell}, \mu^{d}(s)=\sqrt{\ell} s$ and $\mu^{f f}(s)=0$ for $s \geq 0$.

We now show that the second line of (13) holds with $\mu^{d}=0$ if and only if $h_{p}=h_{d}$. 
$(\Leftarrow)$ : By setting $\Psi_{p}=\Psi_{d}$, we see from (69) that $\Delta h_{e}=0$ in (68) and we obtain the desired result by following the reasoning above.

$(\Rightarrow)$ : We proceed by contradiction and suppose $\Psi_{p} \neq \Psi_{d}$ and Assumption 1 holds with $\mu^{d}=0$. Then, according to (13) and since $W(\kappa, e) \leq \sqrt{\ell}|e|$, we know that there exists $\beta \in$ $\mathcal{K} \mathcal{L}$ such that for any $\left(e(0), e_{d}(0), e_{f f}(0)\right) \in \mathbb{R}^{n_{e}+n_{e_{d}}+n_{e_{u}}}$, $\kappa(0) \in \mathbb{Z}_{\geq 0}$, the solutions to $e^{+}=h_{e}\left(\kappa, e, e_{d}, e_{f f}\right)$ satisfy for any $j \in \mathbb{Z}_{>_{0}}:|e(j)| \leq \beta(|e(0)|, j)$, from which we deduce that for $e(0)=0$ and any $\left(e_{d}(0), e_{f f}(0)\right) \in \mathbb{R}^{n_{e_{d}}+n_{e_{u}}}$ and $\kappa(0) \in$ $\mathbb{Z}_{\geq 0},|e(1)|=0$. On the other hand, $\Psi_{p} \neq \Psi_{d}$ means that there exists at least one component of $e_{d}$ denoted $e_{d}^{i}$ that is not assigned to the same node as $e_{p}^{i}$. Without loss of generality, we suppose that $i$ is the only such node. Take $e_{\xi}(0)=0, e_{d}^{k}(0)=0$ if $k \neq i, e_{d}^{i}(0) \neq 0$ which implies that $e_{p}^{k}(0)=0$ if $k \neq i$ and $e_{p}^{i}(0)=e_{d}^{i}(0)$. Consider $e_{f b}(0)=0, e_{f f}(0)=0$ and $\kappa(0)=0$. In view of (64), we have that $e_{\xi}^{+}=\left(\mathbb{I}-\Psi_{p}(\kappa)\right) e_{\xi}+\left(\Psi_{d}(\kappa)-\right.$ $\left.\Psi_{p}(\kappa)\right) e_{d}$ and $e_{f b}^{+}=\left(\mathbb{I}-\Psi_{f b}(\kappa)\right) e_{f b}$. Consequently $e_{\xi}(1)=$ $\left(\mathbb{I}-\Psi_{p}(0)\right) e_{\xi}(0)+\left(\Psi_{d}(0)-\Psi_{p}(0)\right) e_{d}(0)=\left(\Psi_{d}(0)-\Psi_{p}(0)\right)$ $e_{d}(0)$ and $e_{f b}(1)=\left(\mathbb{I}-\Psi_{f b}(0)\right) e_{f b}(0)=0$. Since all the network-induced errors components are initialized at 0 except $e_{p}^{i}(0)$ and $e_{d}^{i}(0)$, we can equivalently assume that either $e_{p}^{i}$ or $e_{d}^{i}$ is reset to 0 at the first transmission instant. We assume that it is $e_{d}^{i}$. In that way, the $i^{\text {th }}$ diagonal component of $\Psi_{d}$ is equal to 1 while the $i^{\text {th }}$ diagonal component of $\Psi_{p}$ is equal to 0 , since $e_{p}^{i}$ and $e_{d}^{i}$ are not associated to the same node. As a consequence, since $\Psi_{p}$ and $\Psi_{d}$ are diagonal matrices and in view of the definition of $e_{d}(0),\left(\Psi_{d}(0)-\Psi_{p}(0)\right) e_{d}(0) \neq 0$. Hence, $e(1) \neq$ 0 which contradicts $|e(1)|=0$. Hence, Assumption 1 only holds with $\mu^{d}=0$ when $\Psi_{p}=\Psi_{d}$, i.e., when $h_{p}=h_{d}$.

\section{REFERENCES}

[1] R. Postoyan, N. van de Wouw, D. Nešić, and W. Heemels, "Emulationbased tracking solutions for nonlinear networked control systems," in Proc. IEEE Conf. Decision Control, 2012, pp. 740-745.

[2] D. Carnevale, A. Teel, and D. Nešić, "A Lyapunov proof of an improved maximum allowable transfer interval for networked control systems," IEEE Trans. Autom. Control, vol. 52, no. 5, pp. 892-897, 2007.

[3] W. Heemels, A. Teel, N. van de Wouw, and D. Nešić, "Networked control systems with communication constraints: Tradeoffs between transmission intervals, delays and performance," IEEE Trans. Autom. Control, vol. 55, no. 8, pp. 1781-1796, 2010.

[4] D. Nešić and A. Teel, "Input-output stability properties of networked control systems," IEEE Trans. Autom. Control, vol. 49, pp. 1650-1667, 2004.

[5] D. Nešić and A. Teel, "Input-to-state stability of networked control systems," Automatica, vol. 40, pp. 2121-2128, 2004.

[6] G. Walsh, O. Beldiman, and L. Bushnell, "Asymptotic behavior of nonlinear networked control systems," IEEE Trans. Autom. Control, vol. 46, pp. 1093-1097, 2001.

[7] H. Gao and T. Chen, "Network-based $H_{\infty}$ output tracking control," IEEE Trans. Autom. Control, vol. 53, no. 3, pp. 655-667, 2008.

[8] N. van de Wouw, P. Naghshtabrizi, M. Cloosterman, and J. Hespanha, "Tracking control for sampled-data systems with uncertain sampling intervals and delays," Int. J. Robust Nonlin. Control, vol. 20, no. 4, pp. 387411, 2010.

[9] S. Yüksel, H. Hindi, and L. Crawford, "Optimal tracking with feedbackfeedforward control separation over a network," in Proc. Amer. Control Conf., Minneapolis, MN, 2006, pp. 3500-3506.

[10] D. Nešić and D. Liberzon, "A unified framework for design and analysis of networked and quantized control systems," IEEE Trans. Autom. Control, vol. 54 , pp. 732-747, 2009.

[11] R. Goebel, R. Sanfelice, and A. Teel, "Hybrid dynamical systems: Robust stability and control for systems that combine continuous-time and discrete-time dynamics," IEEE Control Systems Mag., pp. 28-83, Apr. 2009.

[12] H. Nijmeijer and I. Mareels, "An observer looks at synchronization," IEEE Trans. Circuits Syst. I, vol. 44, no. 10, pp. 882-890, 1997.

[13] R. Postoyan and D. Nešić, "A framework for the observer design for networked control systems," IEEE Trans. Autom. Control, vol. 57, no. 5 , pp. 1309-1314, 2012.

[14] R. Postoyan and D. Nešić, "On emulated nonlinear reduced-order observers for networked control systems," Automatica, vol. 48, pp. 645-652, 2012

[15] T. Ahmed-Ali and F. Lamnabhi-Lagarrigue, "High gain observer design for some networked control systems," IEEE Trans. Autom. Control, vol. 57, no. 4, pp. 995-1000, 2012.

[16] R. Goebel, R. Sanfelice, and A. Teel, Hybrid Dynamical Systems. Princeton, NJ: Princeton Univ. Press, 2012.

[17] C. Cai and A. Teel, "Characterizations of input-to-state stability for hybrid systems," Syst. Control Lett., vol. 58, pp. 47-53, 2009.

[18] M. Tabbara and D. Nešić, "Input-output stability with input-to-state stable protocols for quantized and networked control systems," in Proc. IEEE Conf. Decision Control, Cancun, Mexico, 2008, pp. 2680-2685.

[19] V. Lakshmikantham, V. Matrosov, and S. Sivasundaram, Vector Lyapunov Functions and Stability Analysis of Nonlinear Systems, vol. 63. New York: Springer, 1991

[20] M. Farza, M. M'Saad, and L. Rossignol, "Observer design for a class of MIMO nonlinear systems," Automatica, vol. 40, pp. 135-143, 2004.

[21] J. Gauthier, H. Hammouri, and S. Othman, "A simple observer for nonlinear systems-Applications to bioreactors," IEEE Trans. Autom. Control, vol. 37, pp. 875-880, 1992.

[22] H. Khalil and F. Esfandiari, "Semiglobal stabilization of a class of nonlinear systems using output feedback," IEEE Trans. Autom. Control, vol. 38, no. 9, pp. 1412-1415, 1993.

[23] D. Laila and D. Nešić, "Lyapunov based small-gain theorem for parameterized discrete-time interconnected ISS systems," in Proc. IEEE Conf. Decision Control, Las Vegas, NV, 2002, pp. 2292-2297.

[24] D. Nešić, A. R. Teel, and D. Carnevale, "Explicit computation of the sampling period in emulation of controllers for nonlinear sampled-data systems," IEEE Trans. Autom. Control, vol. 54, no. 3, pp. 619-624, 2009.

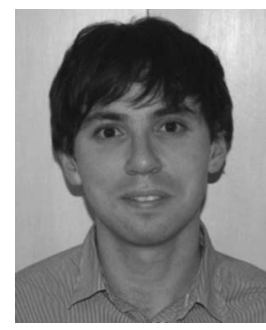

Romain Postoyan received the MSc degree in electrical and control engineering from ENSEEIHT, Toulouse, France, in 2005, the M.Sc. degree in control theory and application from Coventry University, Coventry, U.K., in 2006, and the Ph.D. degree in control theory from Université Paris-Sud, Paris, France, in 2009 .

In 2010, he was a Research Assistant at the University of Melbourne, Melbourne, Australia. Since 2011, he is a CNRS Researcher at the Centre de Recherche en Automatique de Nancy, France.

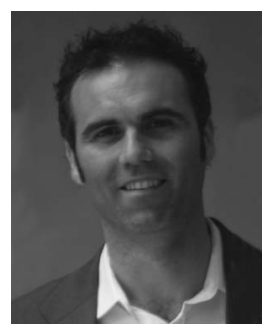

Nathan van de Wouw was born in 1970. He received the M.Sc. degree (with honours) and the $\mathrm{Ph} . \mathrm{D}$. degree in mechanical engineering from the Eindhoven University of Technology, Eindhoven, The Netherlands, in 1994 and 1999, respectively.

From 1999 to 2013, he was affiliated with the Department of Mechanical Engineering, Eindhoven University of Technology in the group of Dynamics and Control as an Assistant/Associate Professor. In 2000, he was with Philips Applied Technologies, Eindhoven, and, in 2001, he was with the Netherlands Organization for Applied Scientific Research (TNO), Delft, The Netherlands. He has held positions as a Visiting Professor at the University of California Santa Barbara, in 2006/2007, at the University of Melbourne, Australia, in 2009/2010 and at the University of Minnesota, Minneapolis, in 2012 and 2013. He has published a large number of journal and conference papers and the books Uniform Output Regulation of Nonlinear Systems: A Convergent Dynamics Approach (Birkhauser, 2005) and Stability and Convergence of Mechanical Systems with Unilateral Constraints (Springer-Verlag, 2008). $\mathrm{He}$ currently is an Associate Editor for the journal Automatica. His current research interests are the analysis and control of nonlinear/non-smooth systems and networked control systems. 


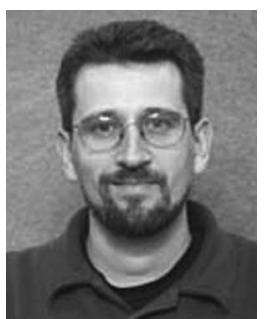

Dragan Nešić (F'08) received the B.E. degree in mechanical engineering from The University of Belgrade, Belgrade, Yugoslavia, in 1990 and the $\mathrm{Ph} . \mathrm{D}$. degree from Systems Engineering, RSISE, Australian National University, Canberra, Australia, in 1997.

Since 1999, he has been with the Department of Electrical and Electronic Engineering (DEEE) of the University of Melbourne, where he currently is a Professor. His research interests include networked control systems, discrete-time, sampled-data and continuous-time nonlinear control systems, input-to-state stability, extremum seeking control, applications of symbolic computation in control theory, hybrid control systems, and so on.

Dr Nešić received the Humboldt Research Fellowship (2003) from the Alexander von Humboldt Foundation, an Australian Professorial Fellowship (2004-2009) and Future Fellowship (2010-2014) from the Australian Research Council. He is a Fellow of IEAust. He is currently a Distinguished Lecturer of CSS, IEEE (2008-). He served as an Associate Editor for Automatica, IEEE Transactions On AUtOMATIC CONTROL, Systems and Control Letters, and the European Journal of Control.

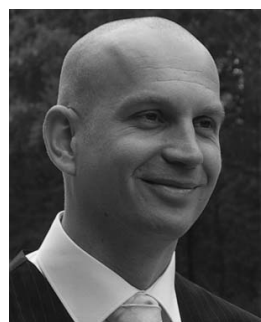

W. P. Maurice H. Heemels (SM'10) received the M.Sc. degree in mathematics and the Ph.D. degree in control theory (both summa cum laude) from the Eindhoven University of Technology (TU/e), Eindhoven, the Netherlands, in 1995 and 1999, respectively.

From 2000 to 2004, he was with the Electrical Engineering Department, TU/e, as an Assistant Professor and from 2004 to 2006 with the Embedded Systems Institute (ESI) as a Research Fellow. Since 2006, he has been with the Department of Mechanical Engineering, TU/e, where he is currently a Full Professor in the Hybrid and Networked Systems Group. He held visiting research positions at the Swiss Federal Institute of Technology (ETH), Zurich, Switzerland (2001) and at the University of California at Santa Barbara (2008). In 2004, he was also at the Research and Development Laboratory, Océ, Venlo, the Netherlands. His current research interests include hybrid and cyber-physical systems, networked and event-triggered control systems and constrained systems including model predictive control. He was an Associate Editor for Nonlinear Analysis: Hybrid Systems, and is currently an Associate Editor for Automatica.

Dr. Heemels served as the General Chair of the 4th IFAC Conference on Analysis and Design of Hybrid Systems (ADHS), Eindhoven, The Netherlands, 2012, and was the IPC Chair for the 4th IFAC Workshop on Distributed Estimation and Control in Networked Systems (NECSYS), Koblenz, Germany, 2013. 